Provided for non-commercial research and education use. Not for reproduction, distribution or commercial use.

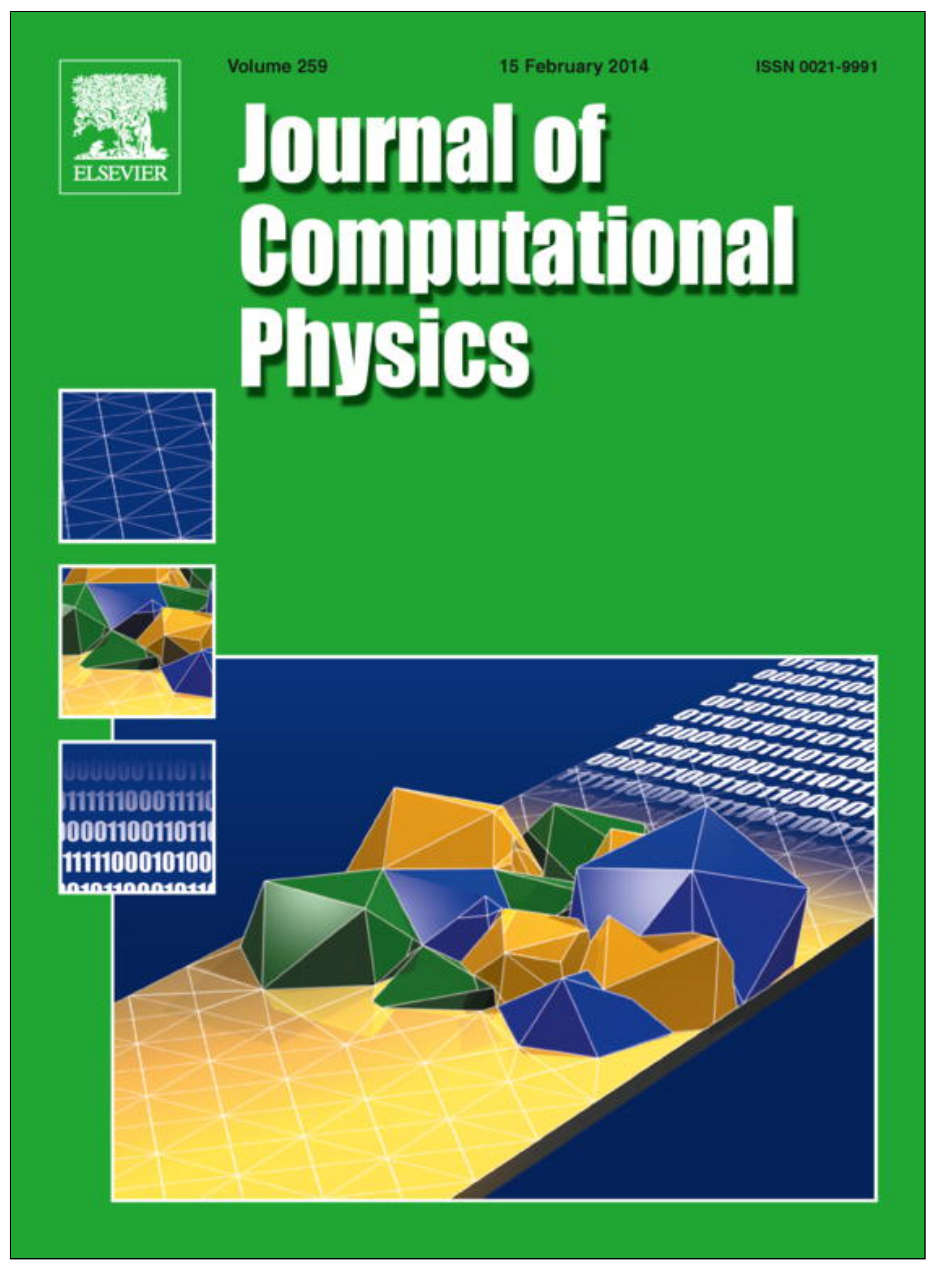

This article appeared in a journal published by Elsevier. The attached copy is furnished to the author for internal non-commercial research and education use, including for instruction at the authors institution and sharing with colleagues.

Other uses, including reproduction and distribution, or selling or licensing copies, or posting to personal, institutional or third party websites are prohibited.

In most cases authors are permitted to post their version of the article (e.g. in Word or Tex form) to their personal website or institutional repository. Authors requiring further information regarding Elsevier's archiving and manuscript policies are encouraged to visit:

http://www.elsevier.com/authorsrights 


\title{
A mixture-energy-consistent six-equation two-phase numerical model for fluids with interfaces, cavitation and evaporation waves
}

\author{
Marica Pelanti ${ }^{\mathrm{a}, *}$, Keh-Ming Shyue ${ }^{\mathrm{b}}$ \\ a Department of Mechanical Engineering, École Nationale Supérieure de Techniques Avancées - ENSTA ParisTech, \\ 828, Boulevard des Maréchaux, 91762 Palaiseau Cedex, France \\ b Department of Mathematics, National Taiwan University, Taipei 106, Taiwan
}

\section{A R T I C L E I N F O}

\section{Article history:}

Received 31 March 2013

Received in revised form 30 October 2013

Accepted 1 December 2013

Available online 10 December 2013

\section{Keywords:}

Multiphase compressible flow models

Mechanical relaxation

Thermo-chemical relaxation

Cavitation

Phase transition

Finite volume schemes

Wave propagation algorithms

Riemann solvers

\begin{abstract}
A B S T R A C T
We model liquid-gas flows with cavitation by a variant of the six-equation single-velocity two-phase model with stiff mechanical relaxation of Saurel-Petitpas-Berry (Saurel et al., 2009) [9]. In our approach we employ phasic total energy equations instead of the phasic internal energy equations of the classical six-equation system. This alternative formulation allows us to easily design a simple numerical method that ensures consistency with mixture total energy conservation at the discrete level and agreement of the relaxed pressure at equilibrium with the correct mixture equation of state. Temperature and Gibbs free energy exchange terms are included in the equations as relaxation terms to model heat and mass transfer and hence liquid-vapor transition. The algorithm uses a high-resolution wave propagation method for the numerical approximation of the homogeneous hyperbolic portion of the model. In two dimensions a fully-discretized scheme based on a hybrid HLLC/Roe Riemann solver is employed. Thermo-chemical terms are handled numerically via a stiff relaxation solver that forces thermodynamic equilibrium at liquid-vapor interfaces under metastable conditions. We present numerical results of sample tests in one and two space dimensions that show the ability of the proposed model to describe cavitation mechanisms and evaporation wave dynamics.
\end{abstract}

(c) 2013 Elsevier Inc. All rights reserved.

\section{Introduction}

The modeling of cavitating flows is relevant in numerous areas of engineering, from naval and submarine systems design to aerospace and nuclear power plants technologies. Cavitating fluids are multiphase mixtures that often involve complex hydrodynamic and thermodynamic processes: liquid-vapor phase transition, dynamical creation of interfaces, vapor structures collapse, and associated shock wave formation and interaction (cf. [1-3]). As a further reason of complexity, in many industrial applications these flows occur in irregular geometries and they have a multi-dimensional character.

Extensive work has been dedicated in the past decades to the simulation of cavitating flows and liquid-vapor flows with phase change, see for instance [4-15] and the references therein. Among the different modeling approaches, the class of hyperbolic compressible multiphase models stemming from the original model of Baer-Nunziato [16] has shown great capabilities in describing the complex wave patterns and thermodynamic mechanisms of cavitation. A first essential feature

\footnotetext{
* Corresponding author. Tel.: +33 1693198 19; fax: +33169319997.

E-mail addresses: marica.pelanti@ensta-paristech.fr (M. Pelanti), shyue@ntu.edu.tw (K.-M. Shyue).
} 
of these models is that compressibility is taken into account for all phases, vapor as well as liquid. This is fundamental to correctly capture wave propagation phenomena and acoustic perturbations, and it is particularly crucial when liquidvapor transition occurs [8]. Another important property is that these models can retain temperature and Gibbs free energy non-equilibrium effects, thus they are able to capture metastable states as well as evaporation fronts, when heat and mass transfer processes are included in the physical description through thermal and chemical relaxation source terms.

There exist various formulations of compressible temperature non-equilibrium multiphase flow models, depending on the assumptions on mechanical and kinetic phase equilibrium. In choosing a particular model, one has to find a good compromise between the accuracy of the description of the physical phenomena and the ability of conceiving robust and efficient numerical methods. In the present work, we are interested in the hyperbolic single-velocity six-equation model proposed by Saurel, Petitpas and Berry in [9] for compressible two-phase flows, see also Zein et al. [17]. This model consists of an advection equation for the volume fraction of one phase, mass and internal energy equations for each phase, and a mixture momentum equation. The six-equation model assumes instantaneous velocity equilibrium between the two phases, but it retains mechanical, thermal and chemical non-equilibrium effects. In the limit of instantaneous pressure relaxation the model reduces to the well known compressible two-phase flow model of Kapila et al. [18]. Nonetheless, as emphasized in [9], and as we briefly recall in Section 2, numerically it is more advantageous to solve the six-equation system with stiff mechanical relaxation rather than the Kapila et al. [18] pressure-equilibrium five-equation model system.

The single-velocity six-equation two-phase model with stiff pressure relaxation was employed in [9] for applications to interface problems and mechanical cavitation processes (that is cavitation with no phase transition). It was later used by Zein et al. in [17] to simulate liquid-vapor transition in metastable liquids. One difficulty of the numerical algorithm illustrated in the latter work, as noted by the authors, is that it may require a very small time step for stability for some expansion problems with phase transition, due to the stiffness of the chemical relaxation terms. Only one-dimensional numerical results are presented by the authors in [17].

The aim of the present paper is to conceive a new multiphase flow computational model on the basis of the six-equation system of [9] that could deal efficiently with interfaces, cavitation and evaporation waves, while retaining simplicity and time-affordability. The key idea of our approach is to employ an alternative mathematical formulation of the standard six-equation model system [9] in the numerical discretization. Rather than using the two phasic internal energy equations of the classical model, in our algorithm we employ two equations for the phasic total energies. Mathematically, these two model systems are equivalent. The present model, however, is numerically advantageous with respect to the standard one, since it allows us to easily design a simple numerical method that ensures important consistency properties with mixture total energy conservation and with the mixture thermodynamic state. More specifically, first, we are able to automatically recover a conservative discrete form of the mixture total energy equation, whereas the classical six-equation model system needs to be augmented with an additional conservation law for the mixture total energy to correct the thermodynamic state [9,17]. Secondly, as a consequence of the mixture total energy conservation consistency property, we are able to easily ensure agreement of the relaxed pressure at equilibrium with the correct mixture equation of state for the full six-equation two-phase model that includes mechanical and thermo-chemical stiff relaxation effects. Relaxation terms are therefore efficiently handled.

To numerically solve the proposed two-phase model with pressure, temperature, and Gibbs free energy relaxation, we employ a simple fractional step approach that consists of the homogeneous hyperbolic system solution step, and a sequence of steps thereafter to solve systems of ordinary differential equations containing the relaxation source terms. A highresolution wave propagation method based on Riemann solvers (HLLC and Roe) (cf. [19]) is employed for the numerical solution of the homogeneous hyperbolic system. The algorithm is easily implemented in the framework of the CLAWPACK software package [20]. For solving the ordinary differential equations with stiff relaxation sources, we have devised robust solvers that drive the mixture to the desired equilibrium conditions in a sequence of relaxation processes (cf. [21,10,8,17, 14]). In this procedure, similar to [8,22], thermodynamic equilibrium is forced at liquid-vapor interfaces under metastable conditions. Numerically for this task we employ an idea similar to [14,23] that uses the thermodynamic equilibrium conditions to reduce the solution of the ODEs relaxation problem to the solution of a simple system of algebraic equations for the equilibrium state variables.

This paper is organized as follows. In Section 2.1, we begin by recalling the six-equation single-velocity model with stiff mechanical relaxation of Saurel-Petitpas-Berry [9] for compressible two-phase flows. We then propose in Section 2.2 a variant of this model system, by employing phasic total energy equations in the mathematical formulation instead of the phasic internal energy equations of the classical approach. The extended model that includes thermal and chemical relaxation terms to model heat and mass transfer is described in Section 2.3. In Section 3 we illustrate the numerical method to solve the basic model system with mechanical relaxation only. In this section we also discuss the mixture-energy-consistency property of the algorithm. The numerical treatment of temperature and Gibbs free energy relaxation source terms is described in Section 4. Finally, in Section 5 we present a selection of numerical results obtained by employing the proposed method with and without activation of heat and mass transfer. 


\section{The six-equation single-velocity two-phase flow model}

\subsection{Phasic-internal-energy-based formulation}

The six-equation single-velocity compressible two-phase flow model with stiff mechanical relaxation proposed by Saurel et al. [9] has the form

$$
\begin{aligned}
& \partial_{t} \alpha_{1}+\vec{u} \cdot \nabla \alpha_{1}=\mu\left(p_{1}-p_{2}\right), \\
& \partial_{t}\left(\alpha_{1} \rho_{1}\right)+\nabla \cdot\left(\alpha_{1} \rho_{1} \vec{u}\right)=0, \\
& \partial_{t}\left(\alpha_{2} \rho_{2}\right)+\nabla \cdot\left(\alpha_{2} \rho_{2} \vec{u}\right)=0 \\
& \partial_{t}(\rho \vec{u})+\nabla \cdot(\rho \vec{u} \otimes \vec{u})+\nabla\left(\alpha_{1} p_{1}+\alpha_{2} p_{2}\right)=0, \\
& \partial_{t}\left(\alpha_{1} \mathcal{E}_{1}\right)+\nabla \cdot\left(\alpha_{1} \mathcal{E}_{1} \vec{u}\right)+\alpha_{1} p_{1} \nabla \cdot \vec{u}=-\mu p_{\mathrm{I}}\left(p_{1}-p_{2}\right), \\
& \partial_{t}\left(\alpha_{2} \mathcal{E}_{2}\right)+\nabla \cdot\left(\alpha_{2} \mathcal{E}_{2} \vec{u}\right)+\alpha_{2} p_{2} \nabla \cdot \vec{u}=\mu p_{\mathrm{I}}\left(p_{1}-p_{2}\right) .
\end{aligned}
$$

Here $\alpha_{k}$ is the volume fraction of phase $k, k=1,2\left(\alpha_{1}+\alpha_{2}=1\right), \rho_{k}$ is the phasic density, $p_{k}$ is the phasic pressure, and $\mathcal{E}_{k}=\rho_{k} \varepsilon_{k}$ is the phasic internal energy, with $\varepsilon_{k}$ denoting the phasic specific internal energy. We have $\rho=\alpha_{1} \rho_{1}+\alpha_{2} \rho_{2}$ denoting the mixture density, and $\vec{u}$ representing the flow velocity vector. The source terms appearing in (1a), (1e), and (1f) model mechanical relaxation. In these terms $\mu>0$ represents the pressure relaxation parameter and $p_{\mathrm{I}}$ is the interface pressure, $p_{\mathrm{I}}=\frac{Z_{2} p_{1}+Z_{1} p_{2}}{Z_{1}+Z_{2}}$, where $Z_{k}=\rho_{k} c_{k}^{2}$ is the acoustic impedance of phase $k$, and $c_{k}$ is the sound speed of phase $k$. We assume an infinite-rate pressure relaxation with $\mu \rightarrow \infty$, therefore mechanical equilibrium is reached instantaneously.

It is known (cf. [9]) that in this instantaneous pressure relaxation limit the six-equation model above reduces to the single-velocity single-pressure five-equation model of Kapila et al. [18] (see also e.g. [24]). The five-equation model system is composed of two phasic mass balance equations, the mixture momentum equation, the mixture total energy equation, and an evolution equation for the volume fraction of one phase with a source term that results from the asymptotic limit of instantaneous velocity and pressure equilibrium of the non-equilibrium compressible two-phase flow model of Baer and Nunziato [16]. Despite this equivalence, the six-equation model (1) offers significant advantages in the numerical approximation, compared to the five-equation model [18]. The main numerical issues in the solution of the five-equation system come from the non-conservative contribution in the volume fraction equation that depends on the divergence of the flow velocity and on the phasic impedances [9,59]. The variation of the volume fraction across acoustic waves associated to this term makes the construction of approximate Riemann solvers more challenging. In particular, the presence of this non-conservative contribution makes it difficult to preserve volume fraction positivity, especially when shocks and strong rarefaction waves are involved [9].

\subsubsection{System's closure}

The closure of system (1) is obtained through the specification of an equation of state for each phase, which we choose to express in terms of $\mathcal{E}_{k}$ and $\rho_{k}, p_{k}=p_{k}\left(\mathcal{E}_{k}, \rho_{k}\right), k=1,2$. The phasic sound speed can be written as $c_{k}=\sqrt{\kappa_{k} h_{k}+\chi_{k}}$, where $h_{k}=\frac{\mathcal{E}_{k}+p_{k}}{\rho_{k}}$ is the phasic specific enthalpy, $\kappa_{k}=\frac{\partial p_{k}\left(\mathcal{E}_{k}, \rho_{k}\right)}{\partial \mathcal{E}_{k}}$ and $\chi_{k}=\frac{\partial p_{k}\left(\mathcal{E}_{k}, \rho_{k}\right)}{\partial \rho_{k}}$. The mixture sound speed of this model is

$$
c=\sqrt{Y_{1} c_{1}^{2}+Y_{2} c_{2}^{2}}
$$

where $Y_{k}=\frac{\alpha_{k} \rho_{k}}{\rho}$ is the mass fraction of phase $k\left(Y_{1}+Y_{2}=1\right)$. Notice the monotonic character of (2) with respect to the volume fraction in contrast to the non-monotonic behavior of the Wood's sound speed [25] of the Kapila et al. model [18]. This feature of the 6-equation model also represents an advantage over the 5-equation model in the numerical approximation. As explained in [26], the non-monotonic behavior of the 5-equation model's sound speed in the numerical diffusion zone of an interface may result in the presence of two sonic points in this region even when the flow is subsonic in the two pure fluids. The construction of approximate Riemann solvers able to handle robustly and efficiently these sonic points does not appear a simple task. In [9] the authors also explain that the sound speed non-monotonicity might affect the propagation of acoustic waves interacting with the interfacial zone, and result in a temporal delay in the wave transmission.

Let us remark that the pressure law $p_{k}=p_{k}\left(\mathcal{E}_{k}, \rho_{k}\right)$, by using a more rigorous terminology, represents an incomplete equation of state. This law suffices to determine the fluid dynamics when thermal and chemical phenomena are neglected, as in the model above. However, a caloric law $T_{k}=T_{k}\left(p_{k}, \rho_{k}\right)$ for each phasic temperature $T_{k}$ is also needed in order to completely describe the thermodynamic state of the fluid (see e.g. [27]). The full thermodynamic characterization is required when heat and mass transfer terms are included in the flow model for problems with phase change, see Section 2.3.

Here we will restrict our study to the case of species governed by the stiffened gas equation of state (SG EOS):

$$
\begin{aligned}
& p_{k}\left(\mathcal{E}_{k}, \rho_{k}\right)=\left(\gamma_{k}-1\right) \mathcal{E}_{k}-\gamma_{k} \pi_{k}-\left(\gamma_{k}-1\right) \eta_{k} \rho_{k}, \\
& T_{k}\left(p_{k}, \rho_{k}\right)=\frac{p_{k}+\pi_{k}}{C_{v k} \rho_{k}\left(\gamma_{k}-1\right)}
\end{aligned}
$$


for $k=1,2$, where $\gamma_{k}, \pi_{k}, \eta_{k}$, and $C_{v k}$ are material-dependent parameters. The associated entropy $s_{k}\left(p_{k}, T_{k}\right)$ and the Gibbs free energy (chemical potential) $g_{k}\left(p_{k}, T_{k}\right)=h_{k}-T_{k} s_{k}$ are given by

$$
\begin{aligned}
& s_{k}\left(p_{k}, T_{k}\right)=C_{v k} \log \frac{T_{k} \gamma_{k}}{\left(p_{k}+\pi_{k}\right)^{\gamma_{k}-1}}+\eta_{k}^{\prime}, \\
& g_{k}\left(p_{k}, T_{k}\right)=\left(\gamma_{k} C_{v k}-\eta_{k}^{\prime}\right) T_{k}-C_{v k} T_{k} \log \frac{T_{k} \gamma_{k}}{\left(p_{k}+\pi_{k}\right)^{\gamma_{k}-1}}+\eta_{k},
\end{aligned}
$$

where $\eta_{k}^{\prime}$ is a constant. With the SG EOS assumption (3), we have $\kappa_{k}=\left(\gamma_{k}-1\right), \chi_{k}=-\left(\gamma_{k}-1\right) \eta_{k}$, and the phasic sound speed can be expressed as $c_{k}=\sqrt{\gamma_{k} \frac{p_{k}+\pi_{k}}{\rho_{k}}}$.

The mixture specific internal energy for the model considered here is defined as $\varepsilon=Y_{1} \varepsilon_{1}+Y_{2} \varepsilon_{2}$, and, equivalently, the mixture internal energy per unit volume is $\mathcal{E}=\rho \varepsilon=\alpha_{1} \mathcal{E}_{1}+\alpha_{2} \mathcal{E}_{2}$. The latter relation, by using the isobaric assumption $p_{1}=p_{2}=p$ in the energy laws $\mathcal{E}_{k}\left(p_{k}, \rho_{k}\right), k=1,2$, gives the mixture equation of state, which determines implicitly the mixture pressure law $p=p\left(\mathcal{E}, \rho_{1}, \rho_{2}, \alpha_{1}\right)$ :

$$
\mathcal{E}=\alpha_{1} \mathcal{E}_{1}\left(p, \rho_{1}\right)+\alpha_{2} \mathcal{E}_{2}\left(p, \rho_{2}\right)
$$

In the case with the SG EOS (3) we find an explicit expression for the mixture pressure $p$ [28]:

$$
p\left(\mathcal{E}, \rho_{1}, \rho_{2}, \alpha_{1}\right)=\frac{\mathcal{E}-\left(\alpha_{1} \rho_{1} \eta_{1}+\alpha_{2} \rho_{2} \eta_{2}\right)-\left(\frac{\alpha_{1} \gamma_{1} \pi_{1}}{\gamma_{1}-1}+\frac{\alpha_{2} \gamma_{2} \pi_{2}}{\gamma_{2}-1}\right)}{\frac{\alpha_{1}}{\gamma_{1}-1}+\frac{\alpha_{2}}{\gamma_{2}-1}} .
$$

When physically relevant values of the flow state variables are defined in the region of thermodynamic stability, the singlevelocity six-equation model (1) is hyperbolic, that is it has real eigenvalues and a complete set of eigenvectors, see for instance [17] and Appendix A. Let us also recall that (1) represents the asymptotic limit of the hyperbolic seven-equation two-velocity two-phase flow model of Saurel and Abgrall [21] for instantaneous kinetic relaxation (see e.g. [17,18]). One advantage of the six-equation model with respect to the two-velocity model is that the order of the eigenvalues is a priori known, yielding an easy decomposition of waves in computing approximate solutions to Riemann problems (cf. [29-31]).

\subsection{Phasic-total-energy-based formulation}

For numerical reasons that we will discuss in the following, we propose to consider a mathematically equivalent formulation of the model (1), obtained by replacing the two phasic internal energy equations with two phasic total energy equations. We denote with $E_{k}=\mathcal{E}_{k}+\frac{1}{2} \rho_{k} \vec{u} \cdot \vec{u}$ the total energy of phase $k$. The alternative form of the six-equation model reads

$$
\begin{aligned}
& \partial_{t} \alpha_{1}+\vec{u} \cdot \nabla \alpha_{1}=\mu\left(p_{1}-p_{2}\right), \\
& \partial_{t}\left(\alpha_{1} \rho_{1}\right)+\nabla \cdot\left(\alpha_{1} \rho_{1} \vec{u}\right)=0, \\
& \partial_{t}\left(\alpha_{2} \rho_{2}\right)+\nabla \cdot\left(\alpha_{2} \rho_{2} \vec{u}\right)=0, \\
& \partial_{t}(\rho \vec{u})+\nabla \cdot(\rho \vec{u} \otimes \vec{u})+\nabla\left(\alpha_{1} p_{1}+\alpha_{2} p_{2}\right)=0, \\
& \partial_{t}\left(\alpha_{1} E_{1}\right)+\nabla \cdot\left(\alpha_{1} E_{1} \vec{u}+\alpha_{1} p_{1} \vec{u}\right)+\Sigma(q, \nabla q)=-\mu p_{\mathrm{I}}\left(p_{1}-p_{2}\right), \\
& \partial_{t}\left(\alpha_{2} E_{2}\right)+\nabla \cdot\left(\alpha_{2} E_{2} \vec{u}+\alpha_{2} p_{2} \vec{u}\right)-\Sigma(q, \nabla q)=\mu p_{\mathrm{I}}\left(p_{1}-p_{2}\right),
\end{aligned}
$$

where the non-conservative term $\Sigma$ appearing in the phasic total energy equations is

$$
\Sigma(q, \nabla q)=-\vec{u} \cdot\left(Y_{2} \nabla\left(\alpha_{1} p_{1}\right)-Y_{1} \nabla\left(\alpha_{2} p_{2}\right)\right)=-\vec{u} \cdot\left(\left(Y_{2} p_{1}+Y_{1} p_{2}\right) \nabla \alpha_{1}+\alpha_{1} Y_{2} \nabla p_{1}-\alpha_{2} Y_{1} \nabla p_{2}\right),
$$

with $q$ denoting the vector of the system unknowns, see (6). Note that unlike the previous model (1) with the phasic internal energy equations, here the simple sum of the two non-conservative phasic total energy equations (6e) and (6f) recovers the equation expressing conservation of the mixture total energy $E=\mathcal{E}+\frac{1}{2} \rho \vec{u} \cdot \vec{u}=\alpha_{1} E_{1}+\alpha_{2} E_{2}$ :

$$
\partial_{t} E+\nabla \cdot\left(E \vec{u}+\alpha_{1} p_{1} \vec{u}+\alpha_{2} p_{2} \vec{u}\right)=0 .
$$

This feature is beneficial in the numerical approximation of the model to ensure consistency with the conservation of $E$, see Section 3.1. 


\subsection{Model with heat and mass transfer}

A classical way to model thermal and chemical inter-phase phenomena mathematically consists in introducing additional heat and mass transfer source terms $\mathcal{Q}$ and $\dot{m}$, respectively, into the original model system (cf. [14,32,21,8,11,17,22]). In the present work we follow in particular the modeling approach of Saurel and co-workers [8,22], employing the six-equation model with phasic total energy equations (6) as the basic system. The flow model augmented with heat and mass transfer terms takes the form:

$$
\begin{aligned}
& \partial_{t} \alpha_{1}+\vec{u} \cdot \nabla \alpha_{1}=\mu\left(p_{1}-p_{2}\right)+\frac{\dot{m}}{\rho_{\mathrm{I}}}, \\
& \partial_{t}\left(\alpha_{1} \rho_{1}\right)+\nabla \cdot\left(\alpha_{1} \rho_{1} \vec{u}\right)=\dot{m}, \\
& \partial_{t}\left(\alpha_{2} \rho_{2}\right)+\nabla \cdot\left(\alpha_{2} \rho_{2} \vec{u}\right)=-\dot{m}, \\
& \partial_{t}(\rho \vec{u})+\nabla \cdot(\rho \vec{u} \otimes \vec{u})+\nabla\left(\alpha_{1} p_{1}+\alpha_{2} p_{2}\right)=0, \\
& \partial_{t}\left(\alpha_{1} E_{1}\right)+\nabla \cdot\left(\alpha_{1} E_{1} \vec{u}+\alpha_{1} p_{1} \vec{u}\right)+\Sigma(q, \nabla q)=-\mu p_{\mathrm{I}}\left(p_{1}-p_{2}\right)+\mathcal{Q}+e_{\mathrm{I}} \dot{m}, \\
& \partial_{t}\left(\alpha_{2} E_{2}\right)+\nabla \cdot\left(\alpha_{2} E_{2} \vec{u}+\alpha_{2} p_{2} \vec{u}\right)-\Sigma(q, \nabla q)=\mu p_{\mathrm{I}}\left(p_{1}-p_{2}\right)-\mathcal{Q}-e_{\mathrm{I}} \dot{m},
\end{aligned}
$$

where the terms $\mathcal{Q}$ and $\dot{m}$ can be written as

$$
\begin{gathered}
\mathcal{Q}=\theta\left(T_{2}-T_{1}\right), \\
\dot{m}=v\left(g_{2}-g_{1}\right) .
\end{gathered}
$$

Here $\theta$ and $v$ are the thermal and chemical relaxation parameters, respectively. These parameters are assumed to be infinite at selected locations, while they are set to zero elsewhere. More specifically, thermal transfer is activated at liquid-vapor interfaces, and thermo-chemical transfer is activated at liquid-vapor interfaces under metastable thermodynamic conditions (liquid temperature $T_{\text {liq }}$ higher than the saturation temperature $T_{\text {sat }}$ at the given pressure):

$$
\begin{aligned}
& \theta= \begin{cases}\infty & \text { if } \epsilon_{\mathrm{I}} \leqslant \alpha_{1} \leqslant 1-\epsilon_{\mathrm{I}}, \\
0 & \text { otherwise }\end{cases} \\
& v= \begin{cases}\infty & \text { if } \epsilon_{\mathrm{I}} \leqslant \alpha_{1} \leqslant 1-\epsilon_{\mathrm{I}} \text { and } T_{\text {liq }}>T_{\text {sat }}, \\
0 & \text { otherwise }\end{cases}
\end{aligned}
$$

Here the parameter $\epsilon_{\mathrm{I}}$ identifies liquid-vapor interface locations (e.g. $\epsilon_{\mathrm{I}}=10^{-4}$ ). See [8] for further discussion on the choice of tolerances.

We refer the reader to [33,34] for a rigorous and systematic analytical study of Liu's subcharacteristic condition [35] for the six-equation two-phase model with the three levels of relaxation considered here, namely, pressure relaxation, simultaneous pressure and temperature relaxation, simultaneous pressure, temperature and chemical potential relaxation. In particular, the works [33,34] show that the subcharacteristic condition (characteristic speeds of the relaxation system at least as large as the characteristic speeds of the relaxed equilibrium system) is satisfied for all cases as long as physical fundamental positivity conditions on a set of physically positive thermodynamic variables are fulfilled.

Let us notice that a theoretical pressure-temperature $(p-T)$ saturation curve can be obtained by imposing the equilibrium condition on the Gibbs free energy $g_{1}=g_{2}$ for the liquid and vapor phases (cf. $\left.[36,37,8]\right)$. With the SG EOS (3a)-(3d), the $p-T$ saturation curve is defined by the equation

$$
A_{\mathrm{S}}+\frac{B_{\mathrm{S}}}{T}+C_{\mathrm{S}} \log T+D_{\mathrm{S}} \log \left(p+\pi_{1}\right)-\log \left(p+\pi_{2}\right)=0
$$

with

$$
A_{\mathrm{S}}=\frac{C_{p 1}-C_{p 2}+\eta_{2}^{\prime}-\eta_{1}^{\prime}}{C_{p 2}-C_{v 2}}, \quad B_{\mathrm{S}}=\frac{\eta_{1}-\eta_{2}}{C_{p 2}-C_{v 2}}, \quad C_{\mathrm{S}}=\frac{C_{p 2}-C_{p 1}}{C_{p 2}-C_{v 2}}, \quad D_{\mathrm{S}}=\frac{C_{p 1}-C_{v 1}}{C_{p 2}-C_{v 2}} .
$$

Here $C_{p k}$ denotes the heat capacity at constant pressure, $C_{p k}=C_{v k} \gamma_{k}, k=1,2$. The parameters in the SG EOS (3a)-(3d) can be chosen to fit the above theoretical $p-T$ curve with the experimental curve for the considered material [36]. The theoretical $p-T$ curve is used in the numerical algorithm, see Section 4.2.

Concerning the interfacial density $\rho_{\mathrm{I}}$ and the interfacial specific total energy $e_{\mathrm{I}}$ that appear in the mass and heat transfer source terms, their expression can be obtained by imposing appropriate thermodynamic constraints on the thermal and chemical processes. For instance, in [17], Zein et al. determine these quantities in a model similar to (8) by assuming pressure and temperature equilibrium during the relaxation processes. However, thanks to the assumption (10) on the relaxation parameters and to the particular numerical algorithm used for the treatment of thermal and chemical source terms, there is no need to specify the expressions of $\rho_{\mathrm{I}}$ and $e_{\mathrm{I}}$ (see Section 4). Let us also note that, due to the symmetry of the source terms in (8), the mixture mass and total energy equations are recovered as for the model in (6). 
To end this section, for the ease of later reference, we write (8) in a compact form as

$$
\partial_{t} q+\nabla \cdot f(q)+\sigma(q, \nabla q)=\psi_{\mu}(q)+\psi_{\theta}(q)+\psi_{\nu}(q)
$$

where

$$
\begin{aligned}
& q=\left(\begin{array}{c}
\alpha_{1} \\
\alpha_{1} \rho_{1} \\
\alpha_{2} \rho_{2} \\
\rho \vec{u} \\
\alpha_{1} E_{1} \\
\alpha_{2} E_{2}
\end{array}\right), \quad f(q)=\left(\begin{array}{c}
0 \\
\alpha_{1} \rho_{1} \vec{u} \\
\alpha_{2} \rho_{2} \vec{u} \\
\rho \vec{u} \otimes \vec{u}+\left(\alpha_{1} p_{1}+\alpha_{2} p_{2}\right) \mathbb{I} \\
\alpha_{1}\left(E_{1}+p_{1}\right) \vec{u} \\
\alpha_{2}\left(E_{2}+p_{2}\right) \vec{u}
\end{array}\right), \quad \sigma(q, \nabla q)=\left(\begin{array}{c}
\vec{u} \cdot \nabla \alpha_{1} \\
0 \\
0 \\
0 \\
\Sigma(q, \nabla q) \\
-\Sigma(q, \nabla q)
\end{array}\right), \\
& \psi_{\mu}(q)=\left(\begin{array}{c}
\mu\left(p_{1}-p_{2}\right) \\
0 \\
0 \\
0 \\
-\mu p_{\mathrm{I}}\left(p_{1}-p_{2}\right) \\
\mu p_{\mathrm{I}}\left(p_{1}-p_{2}\right)
\end{array}\right), \quad \psi_{\theta}(q)=\left(\begin{array}{c}
0 \\
0 \\
0 \\
0 \\
\theta\left(T_{2}-T_{1}\right) \\
-\theta\left(T_{2}-T_{1}\right)
\end{array}\right), \quad \psi_{v}(q)=\left(\begin{array}{c}
v \frac{g_{2}-g_{1}}{\rho_{\mathrm{I}}} \\
v\left(g_{2}-g_{1}\right) \\
-v\left(g_{2}-g_{1}\right) \\
0 \\
v e_{\mathrm{I}}\left(g_{2}-g_{1}\right) \\
-v e_{\mathrm{I}}\left(g_{2}-g_{1}\right)
\end{array}\right)
\end{aligned}
$$

with $\Sigma$ as in (6g). Above we have put into evidence the conservative portion of the spatial derivative contributions in the system as $\nabla \cdot f(q)$, and we have indicated the non-conservative term as $\sigma(q, \nabla q)$. The source terms $\psi_{\mu}(q), \psi_{\theta}(q), \psi_{\nu}(q)$ contain mechanical, thermal and chemical relaxation terms, respectively.

\section{Numerical solution of the model system with mechanical relaxation}

We begin by considering the solution of the model system (12) with only mechanical relaxation:

$$
\partial_{t} q+\nabla \cdot f(q)+\sigma(q, \nabla q)=\psi_{\mu}(q) .
$$

The treatment of thermal and chemical relaxation terms will be described in Section 4. To numerically solve the system above we use a fractional step technique, similar to [9,17], where we alternate between the solution of the homogeneous hyperbolic system and the solution of a system of ordinary differential equations that takes into account pressure relaxation source terms. That is, the algorithm consists of the following steps:

1. Homogeneous hyperbolic system. We solve over a time interval $\Delta t$ the homogeneous hyperbolic portion of (13):

$$
\partial_{t} q+\nabla \cdot f(q)+\sigma(q, \nabla q)=0 .
$$

2. Stiff mechanical relaxation. We solve in the limit $\mu \rightarrow \infty$ the system of ordinary differential equations (ODEs)

$$
\partial_{t} q=\psi_{\mu}(q) .
$$

This step drives the two-phase flow to mechanical equilibrium with an equilibrium relaxed pressure $p_{1}=p_{2}=p$. In this step the partial densities, the mixture momentum, the mixture total energy, and the mixture internal energy remain constant. The volume fraction $\alpha_{1}$, the mixture pressure $p$, and the phasic internal energies $\alpha_{k} \mathcal{E}_{k}, \mathcal{E}_{k}=\mathcal{E}_{k}\left(p,\left(\alpha_{k} \rho_{k}\right) / \alpha_{k}\right)$ for $k=1,2$, are updated before returning to Step 1 .

\subsection{Mixture-energy-consistent discretization}

Before illustrating each step of the algorithm in more details, we emphasize that in the design of a numerical method for computing approximate solutions of the two-phase model (12) it is important to ensure at all times conservation of the quantities that are physically conserved, namely the partial densities $\alpha_{k} \rho_{k}, k=1,2$ (together with the mixture density $\rho=\alpha_{1} \rho_{1}+\alpha_{2} \rho_{2}$ ), the mixture momentum $\rho \vec{u}$, and the mixture total energy $E=\alpha_{1} E_{1}+\alpha_{2} E_{2}$. Note in particular that the values of the equilibrium pressure to be used at the beginning of the homogeneous system solution step must satisfy the mixture EOS (4) for values of $\mathcal{E}=E-\frac{1}{2} \rho \vec{u} \cdot \vec{u}$ that correspond to conservation-consistent discrete values of $E$, so as to approximate correctly the flow thermodynamic state. Godunov-type schemes can be relatively easily formulated to preserve conservation at the discrete level of quantities that are governed by conservation laws (cf. $[38,19]$ ). However, the two-phase mathematical model (either in the form (1) or (6)) does not contain the conservation law for the total energy, but two phasic energy equations from which the total energy is recovered. The difficulty then is to discretize the phasic energy 
equations in such a way that total energy conservation is fulfilled at the discrete level, and that consistency with the correct thermodynamic state is ensured in the sense made precise hereafter.

Let us denote with superscript 0 the quantities computed by solving the homogeneous system in Step 1 of the algorithm above, and with superscript $*$ the quantities at mechanical equilibrium computed in Step 2. As mentioned above (see also Section 3.3), we have $\rho_{k}^{*}=\rho_{k}^{0}, k=1,2,(\rho \vec{u})^{*}=(\rho \vec{u})^{0}, \mathcal{E}^{*}=\mathcal{E}^{0}$, and $E^{*}=E^{0}$. Let us also denote with $E^{0, C}$ discrete values of the mixture total energy that come from a conservative approximation of the conservation law for $E$ in (7).

Definition 3.1. We say that the numerical scheme based on the fractional step algorithm above is mixture-energy-consistent if the following two properties are satisfied

(i) Mixture total energy conservation consistency:

$$
E^{0}=E^{0, C}
$$

where $E^{0}=\left(\alpha_{1} E_{1}\right)^{0}+\left(\alpha_{2} E_{2}\right)^{0}$.

(ii) Relaxed pressure consistency:

$$
\mathcal{E}^{0, \mathrm{C}}=\alpha_{1}^{*} \mathcal{E}_{1}\left(p^{*}, \frac{\left(\alpha_{1} \rho_{1}\right)^{0}}{\alpha_{1}^{*}}\right)+\alpha_{2}^{*} \mathcal{E}_{2}\left(p^{*}, \frac{\left(\alpha_{2} \rho_{2}\right)^{0}}{\alpha_{2}^{*}}\right),
$$

where $\mathcal{E}^{0, \mathrm{C}}=E^{0, \mathrm{C}}-\frac{(\rho \vec{u})^{0} \cdot(\rho \vec{u})^{0}}{2 \rho^{0}}$.

The first property (i) means that the sum of the discrete values of the phasic total energies given by the solution of the homogeneous system must recover discrete values of the mixture total energy that are consistent with a conservative discrete form of (7). The second property (ii) means that the value of the relaxed (equilibrium) pressure $p^{*}$ predicted in the relaxation step must be equal to the pressure as computed through the mixture equation of state $p\left(\mathcal{E}^{0, C}, \alpha_{1}^{*}, \rho_{1}^{*}, \rho_{2}^{*}\right)$, defined by (4). That is, with the SG EOS, this consistency condition reads (cf. (5))

$$
p^{*}=\frac{\mathcal{E}^{0, C}-\left(\left(\alpha_{1} \rho_{1}\right)^{0} \eta_{1}+\left(\alpha_{2} \rho_{2}\right)^{0} \eta_{2}\right)-\left(\frac{\alpha_{1}^{*} \gamma_{1} \pi_{1}}{\gamma_{1}-1}+\frac{\alpha_{2}^{*} \gamma_{2} \pi_{2}}{\gamma_{2}-1}\right)}{\frac{\alpha_{1}^{*}}{\gamma_{1}-1}+\frac{\alpha_{2}^{*}}{\gamma_{2}-1}} .
$$

The mathematical formulation of the two-phase model with the phasic total energy equations (6) easily allows us to satisfy both properties (i) and (ii). To ensure the property (i), it suffices in Step 1 to apply a standard conservative scheme to the conservative portion of the energy equations (6e) and (6f), that is $\partial_{t}\left(\alpha_{k} E_{k}\right)+\nabla \cdot\left(\alpha_{k} E_{k} \vec{u}+\alpha_{k} p_{k} \vec{u}\right), k=1,2$, and to discretize symmetrically the non-conservative contribution $\Sigma$ appearing there. In such a way, the sum of the discrete nonconservative energy equations recovers a conservative discrete form of the mixture energy equation, as a consequence of the cancellation of non-conservative discrete contributions. The fulfillment of mixture total energy conservation consistency then easily enables us to ensure also the property (ii), the agreement of the relaxed equilibrium pressure with the correct mixture equation of state. See the simple pressure relaxation procedure for Step 2 in Section 3.3.

On the other hand, it appears difficult to obtain a mixture-energy-consistent scheme if we apply an analogous fractional step algorithm to the classical six-equation two-phase model (1). Although clearly both formulations (1) and (6) mathematically recover the conservation law for the mixture total energy, it seems hard to discretize the phasic internal energies equations (1e) and (1f) in a way that recovers a conservative discrete form of (7). Indeed, numerical models such as $[9,17]$ built on the formulation (1) need to augment the six-equation system with the equation for $E$. The additional conservation law for $E$ is solved through a standard conservative scheme to obtain consistent discrete values $E^{0, C}$. These values are then used to correct the thermodynamic state predicted by the non-conservative internal energy equations via the mixture equation of state. Note that this approach in general does not guarantee the consistency property (ii), that is $p^{*}=p\left(\mathcal{E}^{0, C}, \alpha_{1}^{*}, \rho_{1}^{*}, \rho_{2}^{*}\right)$.

\subsection{Homogeneous hyperbolic system solution step}

In Step 1 of the algorithm we employ the wave propagation method of [19,39] to compute approximate solutions of the homogeneous system (14). This method belongs to a class of Godunov-type finite volume schemes [40,41,38,19] for solving hyperbolic systems of partial differential equations. We describe hereafter the basic ideas of the method in one dimension. The two-dimensional scheme will be briefly recalled in Section 3.2.4.

\subsubsection{One-dimensional wave-propagation scheme}

We consider the solution of the one dimensional system $\partial_{t} q+\partial_{x} f(q)+\sigma\left(q, \partial_{x} q\right)=0$ (as obtained by setting $\vec{u}=u$ and $\nabla=\partial_{x}$ in (12)). We assume a uniform grid with cells of width $\Delta x$, and we denote with $Q_{i}^{n}$ the approximate solution at the $i$ th cell at time $t^{n}, i \in \mathbb{Z}, n \in \mathbb{N}$. Setting $\Delta t=t^{n+1}-t^{n}$, the second-order one-dimensional wave-propagation scheme has the form 


$$
Q_{i}^{n+1}=Q_{i}^{n}-\frac{\Delta t}{\Delta x}\left(\mathcal{A}^{+} \Delta Q_{i-1 / 2}+\mathcal{A}^{-} \Delta Q_{i+1 / 2}\right)-\frac{\Delta t}{\Delta x}\left(F_{i+1 / 2}^{\mathrm{h}}-F_{i-1 / 2}^{\mathrm{h}}\right),
$$

where $\mathcal{A}^{\mp} \Delta Q_{i+1 / 2}$ are the so-called fluctuations at interfaces $x_{i+1 / 2}$ between cells $i$ and $(i+1)$, and $F_{i+1 / 2}^{\mathrm{h}}$ are second-order correction fluxes for higher resolution.

Here the fluctuations $\mathcal{A}^{\mp} \Delta Q_{i+1 / 2}$ are computed by solving local Riemann problems at cell interfaces $x_{i+1 / 2}$ for each pair of data $Q_{i}^{n}, Q_{i+1}^{n}$ corresponding to adjacent cells. A Riemann solver (cf. $[41,38,19]$ ) must be provided to perform this task.

Let us specify the jump relations that need to be satisfied by an approximate Riemann solver for a Riemann problem with left and right initial data $q_{\ell}$ and $q_{r}$. The Riemann solution structure defined by the solver can be expressed in general by a set of $M$ waves $\mathcal{W}^{l}$ and corresponding speeds $s^{l}, M \gtreqless 6$. For example, see the HLLC-type solver ( $\left.M=3\right)$ and the Roe-type solver $(M=6)$ presented below. By using the formalism introduced in [42], we also define the $f$-waves $\mathcal{Z}^{l}=s^{l} \mathcal{W}^{l}$, $l=1, \ldots, M$, which have the dimension of a flux. The sum of the waves must be equal to the initial jump in the vector $q$ of the system variables:

$$
\Delta q \equiv q_{r}-q_{\ell}=\sum_{l=1}^{M} \mathcal{W}^{l}
$$

Moreover, for any variable of the model system governed by a conservative equation the initial jump in the associated flux function must be recovered by the sum of the associated $f$-wave components. In the considered model the conserved quantities are $\alpha_{k} \rho_{k}, k=1,2$, and $\rho u$, therefore in order to guarantee conservation we need:

$$
\Delta f^{(\xi)} \equiv f^{(\xi)}\left(q_{r}\right)-f^{(\xi)}\left(q_{\ell}\right)=\sum_{l=1}^{M} s^{l} \mathcal{W}_{\xi}^{l}=\sum_{l=1}^{M} \mathcal{Z}_{\xi}^{l}
$$

for $\xi=2,3,4$, where $f^{(\xi)}$ is the $\xi$ th component of the flux vector $f$, and $\mathcal{W}_{\xi}^{l}$ and $\mathcal{Z}_{\xi}^{l}$ denote the $\xi$ th component of the lth wave and of the $l$ th $f$-wave, $l=1, \ldots, M$, respectively. It is clear that conservation of the partial densities ensures conservation of the mixture density $\rho=\alpha_{1} \rho_{1}+\alpha_{2} \rho_{2}$. In addition, we must ensure conservation of the mixture total energy, that is the consistency condition (i) in (16a):

$$
\Delta f_{E} \equiv f_{E}\left(q_{r}\right)-f_{E}\left(q_{\ell}\right)=\sum_{l=1}^{M} s^{l}\left(\mathcal{W}_{5}^{l}+\mathcal{W}_{6}^{l}\right)=\sum_{l=1}^{M} \mathcal{Z}_{5}^{l}+\mathcal{Z}_{6}^{l},
$$

where $f_{E}=u\left(E+\alpha_{1} p_{1}+\alpha_{2} p_{2}\right)$ is the flux function associated to the mixture total energy $E$.

Once the Riemann solution structure $\left\{\mathcal{W}_{i+1 / 2}^{l}, s_{i+1 / 2}^{l}\right\}_{l=1, \ldots, M}$ arising at each cell edge $x_{i+1 / 2}$ is defined through a Riemann solver, the fluctuations $\mathcal{A}^{\mp} \Delta Q_{i+1 / 2}$ and the high-resolution correction fluxes $F_{i+1 / 2}^{\mathrm{h}}$ in (18) are computed as

$$
\begin{gathered}
\mathcal{A}^{ \pm} \Delta Q_{i+1 / 2}=\sum_{l=1}^{M}\left(s_{i+1 / 2}^{l}\right)^{ \pm} \mathcal{W}_{i+1 / 2}^{l}, \\
s^{+}=\max (s, 0), s^{-}=\min (s, 0), \text { and } \\
F_{i+1 / 2}^{\mathrm{h}}=\frac{1}{2} \sum_{l=1}^{M}\left|s_{i+1 / 2}^{l}\right|\left(1-\frac{\Delta t}{\Delta x}\left|s_{i+1 / 2}^{l}\right|\right) \mathcal{W}_{i+1 / 2}^{\mathrm{h}, l},
\end{gathered}
$$

where $\mathcal{W}_{i+1 / 2}^{\mathrm{h}, l}$ are a modified version of $\mathcal{W}_{i+1 / 2}^{l}$ obtained by applying to $\mathcal{W}_{i+1 / 2}^{l}$ a limiter function (cf. [19]).

We present in the next sections two approximate Riemann solvers that we have developed for the model system (12): a HLLC-type solver [43,44,38], similar to the solvers described in [9,17], and a new Roe-type solver [45]. The conservation consistency condition (21) (equivalently (16a)) is easily fulfilled in both cases.

\subsubsection{HLLC-type solver}

To begin with, we define an approximate solver for the two-phase system (14) by applying the idea of the HLLC solver of Toro et al. [44] (see also [38]). One difficulty in designing a HLLC-type solver for the present model is related to the non-conservative character of the phasic energy equations, for which we lack a notion of weak solution in the distributional framework (see for instance on this subject [46-49]). Nonetheless, note that to correctly set the initial thermodynamic state for the solution of the homogeneous system we only require that the sum of the phasic total energies computed at the previous time level fulfill the consistency condition $\left(\alpha_{1} E_{1}\right)^{0}+\left(\alpha_{2} E_{2}\right)^{0}=E^{0, C}$. The individual phasic energies are re-set at the beginning of Step 1 by using the relaxed variables $p^{*}$ and $\alpha_{1}^{*}$, obtained in Step 2 through a procedure that by construction ensures that the energies' sum recovers the correct mixture energy state (Section 3.3). The individual phasic energies values that come from the solution of the homogeneous system have only a role in the initial condition for the mechanical relaxation step. Aiming at designing the simplest method that could provide reasonable mixture-energy-consistent estimates for 
$\left(\alpha_{k} E_{k}\right)^{0}$, our first approach consists in simply neglecting the non-conservative contribution $\Sigma\left(q, \partial_{x} q\right)$ in $\partial_{t} q+\partial_{x} f(q)+\sigma=0$. We then apply the standard HLLC method to the conservative portion of the system plus the advection equation for $\alpha_{1}$. The resulting solver consists of three waves $\mathcal{W}^{l}, l=1,2,3$, moving at speeds

$$
s^{1}=S_{\ell}, \quad s^{2}=S^{\star}, \quad \text { and } \quad s^{3}=S_{r}
$$

that separate four constant states $q_{\ell}, q_{\star \ell}, q_{\star r}$ and $q_{r}$. Here we indicate with subscripts $\star \ell, \star r$ quantities corresponding to the states $q_{\star \ell}$ and $q_{\star r}$ adjacent (respectively on the left and on the right) to the middle wave propagating at speed $S^{\star}$. Following Davis [50] we define

$$
S_{\ell}=\min \left(u_{\ell}-c_{\ell}, u_{r}-c_{r}\right) \text { and } S_{r}=\max \left(u_{\ell}+c_{\ell}, u_{r}+c_{r}\right)
$$

The speed $S^{\star}$ is then determined as (see [38])

$$
S^{\star}=\frac{p_{r}-p_{\ell}+\rho_{\ell} u_{\ell}\left(S_{\ell}-u_{\ell}\right)-\rho_{r} u_{r}\left(S_{r}-u_{r}\right)}{\rho_{\ell}\left(S_{\ell}-u_{\ell}\right)-\rho_{r}\left(S_{r}-u_{r}\right)}
$$

The middle states $q_{\star \ell}, q_{\star r}$ are defined so as to satisfy the following Rankine-Hugoniot conditions, based on the conservative portion of the system:

$$
\begin{aligned}
& f^{(\xi)}\left(q_{r}\right)-f^{(\xi)}\left(q_{\star r}\right)=S_{r}\left(q_{r}-q_{\star r}\right), \\
& f^{(\xi)}\left(q_{\star \ell}\right)-f^{(\xi)}\left(q_{\ell}\right)=S_{\ell}\left(q_{\star \ell}-q_{\ell}\right), \\
& f^{(\xi)}\left(q_{\star r}\right)-f^{(\xi)}\left(q_{\star \ell}\right)=S^{\star}\left(q_{\star r}-q_{\star \ell}\right),
\end{aligned}
$$

$\xi=2, \ldots, 6$. Then, the middle states are obtained as

$$
q_{\star \iota}=\left\{\begin{array}{c}
\alpha_{1, \iota} \\
\left(\alpha_{1} \rho_{1}\right)_{\iota} \frac{S_{\iota}-u_{\iota}}{S_{\iota}-S^{\star}} \\
\left(\alpha_{2} \rho_{2}\right)_{\iota} \frac{S_{\iota}-u_{\iota}}{S_{\iota}-S^{\star}} \\
\rho_{\iota} \frac{S_{\iota}-u_{\iota}}{S_{\iota}-S^{\star}} S^{\star} \\
\left(\alpha_{1} \rho_{1}\right)_{\iota} \frac{S_{\iota}-u_{\iota}}{S_{\iota}-S^{\star}}\left(\frac{E_{1, l}}{\rho_{1, l}}+\left(S^{\star}-u_{\iota}\right)\left(S^{\star}+\frac{p_{\iota}}{\rho_{1, \iota}\left(S_{\iota}-u_{\iota}\right)}\right)\right) \\
\left(\alpha_{2} \rho_{2}\right)_{\iota} \frac{S_{l}-u_{\iota}}{S_{\iota}-S^{\star}}\left(\frac{E_{2, l}}{\rho_{2, l}}+\left(S^{\star}-u_{\iota}\right)\left(S^{\star}+\frac{p_{\iota}}{\rho_{2, l}\left(S_{\iota}-u_{\iota}\right)}\right)\right)
\end{array}\right\},
$$

$\iota=\ell, r$. The waves for this simple HLLC-type solver are

$$
\mathcal{W}^{1}=q_{\star \ell}-q_{\ell}, \quad \mathcal{W}^{2}=q_{\star r}-q_{\star \ell}, \quad \text { and } \quad \mathcal{W}^{3}=q_{r}-q_{\star r}
$$

Note that the conditions (27) above for $\xi=2,3,4$ imply the conservation conditions for $\alpha_{k} \rho_{k}$ and $\rho u$ in (20) (with $M=3$ ). For $\xi=5,6$, the conditions (27) imply analogous relations for the conservative portion of the phasic energy equations:

$$
\Delta f^{(\xi)} \equiv f^{(\xi)}\left(q_{r}\right)-f^{(\xi)}\left(q_{\ell}\right)=\sum_{l=1}^{3} s^{l} \mathcal{W}_{\xi}^{l}=\sum_{l=1}^{3} \mathcal{Z}_{\xi}^{l}
$$

for $\xi=5,6$, where $\Delta f^{(4+k)}=u \alpha_{k}\left(E_{k}+p_{k}\right), k=1$, 2. Since $f_{E}(q)=f^{(5)}(q)+f^{(6)}(q)$, the above relations clearly guarantee the consistency condition (21) (equivalently (16a)) with mixture total energy conservation.

Let us mention that it is possible to construct more sophisticated HLLC-type solvers that take into account the nonconservative terms in the phasic energy equations. The idea is to introduce a correction $\Phi^{l}$ to the $f$-wave components $\mathcal{Z}_{5}^{l}$, $\mathcal{Z}_{6}^{l}$ corresponding to the phasic energies to model non-conservative energy exchanges: $\mathcal{Z}_{4+k}^{\Sigma, l}=\mathcal{Z}_{4+k}^{l}+(-1)^{k} \Phi^{l}, k=1,2$, $l=1,2,3$. Note that for any choice of $\Phi^{l}$ we still guarantee the mixture total energy conservation consistency condition (21), thanks to the symmetry of the $f$-wave corrections for the two phasic energies $\alpha_{k} E_{k}, k=1,2$. In fact, in the sum of the mixture total energy flux contributions the terms $\Phi^{l}$ cancel out: $\Delta f_{E}=\Delta f^{(5)}+\Delta f^{(6)}=\sum_{l=1}^{3}\left(\mathcal{Z}_{5}^{\Sigma, l}+\mathcal{Z}_{6}^{\Sigma, l}\right)=\sum_{l=1}^{3}\left(\mathcal{Z}_{5}^{l}+\mathcal{Z}_{6}^{l}\right)$.

We have numerically investigated versions of the HLLC-type solver with different treatment of the non-conservative terms, and no remarkable difference has been observed between the sets of results. Therefore, we choose in general to adopt the simplest method that neglects the $\pm \Sigma$ terms. Extensive numerical experimentation has shown the effectiveness of this approach, based on the results available in the literature. 


\subsubsection{Roe-type solver}

Following the classical Roe's approach [45] (see also [41,38,19]), we define an approximate Riemann solver for (14) by using the exact solution to the Riemann problem for a linearized system $\partial_{t} q+\tilde{A}\left(q_{\ell}, q_{r}\right) \partial_{x} q=0$. The constant coefficient matrix $\tilde{A}=\tilde{A}\left(q_{\ell}, q_{r}\right) \in \mathbb{R}^{6 \times 6}$ (Roe matrix) is an averaged version of the matrix $A(q)$ of the homogeneous model system written in quasi-linear form, $\partial_{t} q+\partial_{x} f(q)+\sigma\left(q, \partial_{x} q\right)=\partial_{t} q+A(q) \partial_{x} q=0$. Note that, in contrast with the HLLC-type method described above, here we include the non-conservative terms $\pm \Sigma\left(q, \partial_{x} q\right)$ in the discretization. The numerical scheme then implicitly defines a meaning for these non-conservative contributions, whose suitability remains nonetheless hard to evaluate.

The choice of the Roe matrix $\tilde{A}=\tilde{A}\left(q_{\ell}, q_{r}\right)$ must guarantee conservation for the quantities that are physically conserved, the partial densities $\alpha_{k} \rho_{k}, k=1,2$, the mixture momentum $\rho u$, and the mixture total energy $E$ (conditions (20) and (21)). That is, denoting with $q^{(\xi)}$ the $\xi$ th component of $q$, and with $f^{(\xi)}$ the associated flux function $(\xi$ th component of $f(q))$, we require:

$$
\sum_{l=1}^{6} \tilde{A}_{\xi l}\left(q_{r}-q_{\ell}\right)^{(l)}=f^{(\xi)}\left(q_{r}\right)-f^{(\xi)}\left(q_{\ell}\right)
$$

for $\xi=2,3,4$, and

$$
\sum_{l=1}^{6}\left(\tilde{A}_{5 l}+\tilde{A}_{6 l}\right)\left(q_{r}-q_{\ell}\right)^{(l)}=f_{E}\left(q_{r}\right)-f_{E}\left(q_{\ell}\right),
$$

where $\tilde{A}_{\xi l}$ is the entry $(\xi, l)$ of the matrix $\tilde{A}$, and $f_{E}=u\left(E+\alpha_{1} p_{1}+\alpha_{2} p_{2}\right)$ is the flux function for the total energy $E$, see Eq. (7). The relations above are equivalent to the conditions (20) and (21). The Riemann solution structure of the Roe solver consists of $M=6$ waves and speeds that correspond to the eigenstructure of the Roe matrix. Denoting with $\tilde{r}_{\xi}$ the (right) Roe eigenvectors, and with $\tilde{\lambda}_{\xi}$ the Roe eigenvalues, we have

$$
\mathcal{W}^{\xi}=\tilde{\beta}_{\xi} \tilde{r}_{\xi} \quad \text { and } \quad s^{\xi}=\tilde{\lambda}_{\xi}, \quad \xi=1, \ldots, 6,
$$

where $\tilde{\beta}_{\xi}$ are the coefficients of the projection of the jump $q_{r}-q_{\ell}$ onto the basis of the Roe eigenvectors, $q_{r}-q_{\ell}=$ $\sum_{\xi=1}^{6} \tilde{\beta}_{\xi} \tilde{r}_{\xi}$. The definition of the Roe matrix and of the Roe eigenstructure for the case of the stiffened gas EOS is reported in Appendix B.

Numerical experiments show agreement of the results obtained with the HLLC-type solver and the Roe-type solver [51]. Let us remark that the HLLC method, as expected, is more robust than the Roe method, and it is the method that we usually prefer to employ for the one-dimensional scheme and for the computation of normal waves in the two-dimensional scheme. The Roe eigenstructure is nonetheless useful in the two-dimensional wave propagation algorithm to define transverse fluctuations [19]. This is done by projecting the normal fluctuations obtained via the HLLC solver onto the basis of Roe eigenvectors associated to the orthogonal direction.

\subsubsection{Two-dimensional wave-propagation scheme}

We consider the solution of system (12) in two dimensions on a Cartesian grid with cells of size $\Delta x$ and $\Delta y$. We denote by $Q_{i j}^{n}$ the approximate solution of the system at the cell $(i, j)$ and at time $t^{n}$. The two-dimensional high-resolution wave propagation algorithm [39,19] has the form

$$
\begin{aligned}
Q_{i j}^{n+1}= & Q_{i j}^{n}-\frac{\Delta t}{\Delta x}\left(\mathcal{A}^{+} \Delta Q_{i-1 / 2, j}+\mathcal{A}^{-} \Delta Q_{i+1 / 2, j}\right)-\frac{\Delta t}{\Delta y}\left(\mathcal{B}^{+} \Delta Q_{i, j-1 / 2}+\mathcal{B}^{-} \Delta Q_{i, j+1 / 2}\right) \\
& -\frac{\Delta t}{\Delta x}\left(F_{i+1 / 2, j}^{\mathrm{h}}-F_{i-1 / 2, j}^{\mathrm{h}}\right)-\frac{\Delta t}{\Delta y}\left(G_{i, j+1 / 2}^{\mathrm{h}}-G_{i, j-1 / 2}^{\mathrm{h}}\right) .
\end{aligned}
$$

Here $\mathcal{A}^{ \pm} \Delta Q$ and $\mathcal{B}^{ \pm} \Delta Q$ are the fluctuations arising from plane-wave Riemann problems in the $x$ and $y$ directions [39]. The fluxes $F_{i+1 / 2, j}^{\mathrm{h}}, G_{i, j+1 / 2}^{\mathrm{h}}$ contain contributions of transverse fluctuations and second-order corrections terms and they are employed to obtain higher accuracy.

The transverse fluctuations are computed by decomposing the fluctuations $\mathcal{A}^{ \pm} \Delta Q$ and $\mathcal{B}^{ \pm} \Delta Q$ into transverse waves. In our scheme we use the eigenstructure of the Roe matrices of the local plane-wave Riemann problems (see Appendix B) to perform the transverse splitting.

The algorithm (33) above can be generalized in a straightforward manner to logically rectangular quadrilateral grids (curvilinear grids) to perform computations in irregularly-shaped domains. We refer the reader to [19,39] for an exhaustive presentation of multi-dimensional wave-propagation algorithms, and to [52,15] for applications to compressible multiphase flow. 


\subsection{Stiff mechanical relaxation step}

In Step 2 of the algorithm the phasic pressures obtained from Step 1 are relaxed to an equilibrium value. We use a procedure similar to the relaxation techniques described in [21,9], in that we consider the system of ODEs (15) and we look for its solution in the limit $\mu \rightarrow \infty$ :

$$
\begin{aligned}
& \partial_{t} \alpha_{1}=\mu\left(p_{1}-p_{2}\right), \\
& \partial_{t}\left(\alpha_{1} \rho_{1}\right)=0, \\
& \partial_{t}\left(\alpha_{2} \rho_{2}\right)=0, \\
& \partial_{t}(\rho \vec{u})=0, \\
& \partial_{t}\left(\alpha_{1} E_{1}\right)=-\mu p_{\mathrm{I}}\left(p_{1}-p_{2}\right), \\
& \partial_{t}\left(\alpha_{2} E_{2}\right)=\mu p_{\mathrm{I}}\left(p_{1}-p_{2}\right) .
\end{aligned}
$$

Recall that the quantities with superscript 0 are those coming from the solution of the homogeneous system in Step 1, and that the quantities with superscript $*$ represent the solution at mechanical equilibrium. As earlier anticipated, from (34b) and $(34 \mathrm{c})$ it is easy to observe that

$$
\left(\alpha_{k} \rho_{k}\right)^{*}=\left(\alpha_{k} \rho_{k}\right)^{0}, \quad k=1,2, \quad \text { and } \quad(\rho \vec{u})^{*}=(\rho \vec{u})^{0},
$$

this yielding also $\rho^{*}=\rho^{0}$ and $\vec{u}^{*}=\vec{u}^{0}$. Now, combining (34a), (34e), (34f), and using (35), we find easily

$$
\begin{aligned}
& \partial_{t}\left(\alpha_{1} E_{1}\right)=\partial_{t}\left(\alpha_{1} \mathcal{E}_{1}\right)=-p_{\mathrm{I}} \partial_{t} \alpha_{1}, \\
& \partial_{t}\left(\alpha_{2} E_{2}\right)=\partial_{t}\left(\alpha_{2} \mathcal{E}_{2}\right)=p_{\mathrm{I}} \partial_{t} \alpha_{1} .
\end{aligned}
$$

Note that the sum of these equations gives $\partial_{t} E=\partial_{t} \mathcal{E}=0$, and hence $E^{*}=E^{0}$ and $\mathcal{E}^{*}=\mathcal{E}^{0}$, meaning that the total energy and the total internal energy of the two-phase mixture do not vary as the phasic pressures relax toward the equilibrium value $p^{*}$.

We introduce now an approximation, by assuming a linear variation of the interface pressure $p_{\mathrm{I}}$ with $\alpha_{1}$ :

$$
p_{\mathrm{I}}=p_{\mathrm{I}}^{0}+\frac{p_{\mathrm{I}}^{*}-p_{\mathrm{I}}^{0}}{\alpha_{1}^{*}-\alpha_{1}^{0}}\left(\alpha_{1}-\alpha_{1}^{0}\right)
$$

With this assumption, the system (36) can be easily integrated, and we obtain

$$
\begin{aligned}
& \left(\alpha_{1} E_{1}\right)^{*}-\left(\alpha_{1} E_{1}\right)^{0}=\left(\alpha_{1} \mathcal{E}_{1}\right)^{*}-\left(\alpha_{1} \mathcal{E}_{1}\right)^{0}=-\frac{p_{\mathrm{I}}^{0}+p_{\mathrm{I}}^{*}}{2}\left(\alpha_{1}^{*}-\alpha_{1}^{0}\right), \\
& \left(\alpha_{2} E_{2}\right)^{*}-\left(\alpha_{2} E_{2}\right)^{0}=\left(\alpha_{2} \mathcal{E}_{2}\right)^{*}-\left(\alpha_{2} \mathcal{E}_{2}\right)^{0}=\frac{p_{\mathrm{I}}^{0}+p_{\mathrm{I}}^{*}}{2}\left(\alpha_{1}^{*}-\alpha_{1}^{0}\right) .
\end{aligned}
$$

Note that the hypothesis (37) is equivalent to approximating $p_{\mathrm{I}}$ in (36) as $p_{\mathrm{I}}=\frac{p_{\mathrm{I}}^{0}+p_{\mathrm{I}}^{*}}{2}$ (an approximation also suggested in [17]).

Next, we impose mechanical equilibrium $p_{1}^{*}=p_{2}^{*}=p_{I}^{*}=p^{*}$, which in particular amounts to express the phasic internal energies at final time as $\mathcal{E}_{k}^{*}=\mathcal{E}_{k}\left(p^{*},\left(\alpha_{k} \rho_{k}\right)^{0} / \alpha_{k}^{*}\right)$ for $k=1,2$. With these relations, together with (35) and the saturation condition $\alpha_{1}+\alpha_{2}=1$, system (38) gives two equations for the two unknowns $\alpha_{1}^{*}$ and $p^{*}$. For the particular case of the SG EOS (3) we can obtain a simple quadratic equation for the relaxed pressure $p^{*}$ (which has always a physically admissible solution) and then easily compute the relaxed volume fraction $\alpha_{1}^{*}$. See Appendix C.1.

Let us remark that the values $p^{*}$ and $\alpha_{1}^{*}$ computed through this algorithm by construction satisfy the mixture internal energy equation (4), hence the relaxed pressure $p^{*}$ verifies the mixture pressure law $p^{*}=p\left(\mathcal{E}^{0}, \alpha_{1}^{*}, \frac{\left(\alpha_{1} \rho_{1}\right)^{0}}{\alpha_{1}^{*}}, \frac{\left(\alpha_{2} \rho_{2}\right)^{0}}{\alpha_{2}^{*}}\right)$, where we have used $\mathcal{E}^{*}=\mathcal{E}^{0}$ and $\left(\alpha_{k} \rho_{k}\right)^{*}=\left(\alpha_{k} \rho_{k}\right)^{0}$. Since the wave propagation scheme that we employ in Step 1 recovers conservation-consistent discrete values of the mixture total energy from the computed phasic energies, $E^{0}=$ $\left(\alpha_{1} E_{1}\right)^{0}+\left(\alpha_{2} E_{2}\right)^{0}=E^{0, C}$, then the computed values of $\mathcal{E}^{0}=E^{0}-\frac{1}{2} \frac{(\rho \vec{u})^{0} \cdot(\rho \vec{u})^{0}}{\rho^{0}}$ also agree with mixture total energy conservation. Based on this observation we deduce that the simple pressure relaxation procedure described here computes the thermodynamically correct value of the equilibrium pressure $p^{*}$. Both properties (i) and (ii) of Definition 3.1 are ensured, and therefore the numerical scheme is mixture-energy-consistent. 


\section{Numerical solution of the extended model system with thermal and chemical relaxation}

We consider now the numerical treatment of heat and mass transfer terms to solve the full model (12). As in [17], we assume that the mechanical relaxation characteristic time $1 / \mu$ is much smaller than the characteristic time scales $1 / \theta, 1 / \nu$ of heat and mass transfer, and that thermal and chemical relaxation occur in conditions of pressure equilibrium. Based on this, heat and mass transfer source terms are handled after solving the system with mechanical relaxation (15). A third step is then added to the fractional step algorithm described in Section 3. This Step 3 of the algorithm is activated at liquid-vapor interfaces only and it includes two sub-steps:

3a. Thermal relaxation. We solve in the limit $\mu \rightarrow \infty, \theta \rightarrow \infty$ the system of ODEs

$$
\partial_{t} q=\psi_{\mu}(q)+\psi_{\theta}(q) .
$$

This sub-step relaxes the phasic temperatures to an equilibrium value $T_{1}=T_{2} \equiv T$ under the constraint of mechanical equilibrium, represented by the stiff pressure relaxation term $\psi_{\mu}(q)$ in the system above. The updated values $T, p$ of the temperature and of the pressure computed through this step are used to check the metastability condition $T_{\text {liq }}=T>T_{\text {sat }}(p)$. If the liquid has reached a metastable state, then mass transfer is also activated by applying the next sub-step $3 \mathrm{~b}$. Otherwise, we update the volume fraction, the pressure and the phasic energies and we return to the solution of the homogeneous system (Step 1).

3b. Thermo-chemical relaxation. If the metastability condition is fulfilled, the system of ODES

$$
\partial_{t} q=\psi_{\mu}(q)+\psi_{\theta}(q)+\psi_{\nu}(q)
$$

is solved in the limit $\mu \rightarrow \infty, \theta \rightarrow \infty, v \rightarrow \infty$. This step of the algorithm relaxes the phasic temperatures and Gibbs free energies to equilibrium values $T_{1}=T_{2} \equiv T$ and $g_{1}=g_{2}$ at liquid-vapor interfaces, while keeping mechanical equilibrium $p_{1}=p_{2}$. Note that in this step the mixture density, momentum, total energy and internal energy remain constant, whereas the partial densities vary. The solution of the system above gives updated values for the volume fraction $\alpha_{1}$, the mixture pressure $p$, the partial densities $\alpha_{k} \rho_{k}$, and the phasic energies $\alpha_{k} \mathcal{E}_{k}, \mathcal{E}_{k}=\mathcal{E}_{k}\left(p,\left(\alpha_{k} \rho_{k}\right) / \alpha_{k}\right)$ before returning to Step 1 .

In the section devoted to the illustration of numerical experiments (Section 5) we will sometimes refer to the thermochemical solver described here as $p-p T-p T g$, this nomenclature representing Steps 2, 3a, and $3 \mathrm{~b}$ of the algorithm.

\subsection{Thermal relaxation step}

We illustrate here the solver used for the temperature relaxation step (Step 3a). We consider the solution of the system of ODEs (39) in the limit $\mu \rightarrow \infty, \theta \rightarrow \infty$ :

$$
\begin{aligned}
& \partial_{t} \alpha_{1}=\mu\left(p_{1}-p_{2}\right), \\
& \partial_{t}\left(\alpha_{1} \rho_{1}\right)=0, \\
& \partial_{t}\left(\alpha_{2} \rho_{2}\right)=0, \\
& \partial_{t}(\rho \vec{u})=0, \\
& \partial_{t}\left(\alpha_{1} E_{1}\right)=-\mu p_{\mathrm{I}}\left(p_{1}-p_{2}\right)+\theta\left(T_{2}-T_{1}\right), \\
& \partial_{t}\left(\alpha_{2} E_{2}\right)=\mu p_{\mathrm{I}}\left(p_{1}-p_{2}\right)-\theta\left(T_{2}-T_{1}\right) .
\end{aligned}
$$

The quantities at initial time are those coming form the pressure relaxation step, denoted with superscript $*$. We indicate with superscript $* *$ the quantities at final time (mechanical and thermal equilibrium). We easily see that the partial densities, the mixture momentum, and the mixture internal and total energy remain constant in this step. Therefore, as in Step 2 of the algorithm, we have

$$
\left(\alpha_{k} \rho_{k}\right)^{* *}=\left(\alpha_{k} \rho_{k}\right)^{0}, \quad k=1,2, \quad(\rho \vec{u})^{* *}=(\rho \vec{u})^{0}, \quad \mathcal{E}^{* *}=\mathcal{E}^{0}, \quad E^{* *}=E^{0} .
$$

The mechanical and thermal equilibrium conditions to be imposed here are

$$
\begin{aligned}
& p_{1}^{* *}=p_{2}^{* *} \equiv p^{* *}, \\
& T_{1}^{* *}=T_{2}^{* *} \equiv T^{* *} .
\end{aligned}
$$

Combining (42) and (43) gives an algebraic system for the unknowns $\alpha_{1}^{* *}, \rho_{k}^{* *}, p^{* *}, T^{* *}$. For the stiffened gas EOS this system can be reduced to the solution of a quadratic equation to be solved for the pressure $p^{* *}$. See Appendix C.2. 


\subsection{Thermo-chemical relaxation step}

We finally describe the solution technique for the temperature and Gibbs free energy relaxation step (Step $3 b$ ). This step is activated if metastable states are reached, $T^{* *}>T_{\text {sat }}\left(p^{* *}\right)$. The saturation temperature $T_{\text {sat }}$ is computed by solving the nonlinear $p-T$ equation in (11) by using Newton's iterative method. We look for the solution of the system of ODEs (40) in the limit $\mu \rightarrow \infty, \theta \rightarrow \infty, v \rightarrow \infty$ :

$$
\begin{aligned}
& \partial_{t} \alpha_{1}=\mu\left(p_{1}-p_{2}\right)+v \frac{g_{2}-g_{1}}{\rho_{\mathrm{I}}}, \\
& \partial_{t}\left(\alpha_{1} \rho_{1}\right)=v\left(g_{2}-g_{1}\right), \\
& \partial_{t}\left(\alpha_{2} \rho_{2}\right)=-v\left(g_{2}-g_{1}\right), \\
& \partial_{t}(\rho \vec{u})=0, \\
& \partial_{t}\left(\alpha_{1} E_{1}\right)=-\mu p_{\mathrm{I}}\left(p_{1}-p_{2}\right)+\theta\left(T_{2}-T_{1}\right)+v e_{\mathrm{I}}\left(g_{2}-g_{1}\right), \\
& \partial_{t}\left(\alpha_{2} E_{2}\right)=\mu p_{\mathrm{I}}\left(p_{1}-p_{2}\right)-\theta\left(T_{2}-T_{1}\right)-v e_{\mathrm{I}}\left(g_{2}-g_{1}\right) .
\end{aligned}
$$

The quantities at initial time are those coming from the mechanical and thermal relaxation step, denoted with superscript $* *$. We denote with superscript $\circledast$ the quantities at final time (mechanical, thermal, and chemical equilibrium). We easily see that the mixture density, momentum, internal energy and total energy remain constant in this step. Therefore, by using the relations (42):

$$
\rho^{\circledast}=\rho^{0}, \quad(\rho \vec{u})^{\circledast}=(\rho \vec{u})^{0}, \quad \mathcal{E}^{\circledast}=\mathcal{E}^{0}, \quad E^{\circledast}=E^{0} .
$$

Hence we can write the mass and mixture energy conservation conditions:

$$
\begin{aligned}
& \rho^{0}=\alpha_{1}^{\circledast} \rho_{1}^{\circledast}+\alpha_{2}^{\circledast} \rho_{2}^{\circledast}, \\
& \mathcal{E}^{0}=\alpha_{1}^{\circledast} \mathcal{E}_{1}^{\circledast}+\alpha_{2}^{\circledast} \mathcal{E}_{2}^{\circledast} .
\end{aligned}
$$

The mechanical, thermal and chemical equilibrium conditions are

$$
\begin{aligned}
& p_{1}^{\circledast}=p_{2}^{\circledast} \equiv p^{\circledast}, \\
& T_{1}^{\circledast}=T_{2}^{\circledast} \equiv T^{\circledast}, \\
& g_{1}^{\circledast}=g_{2}^{\circledast} .
\end{aligned}
$$

Now Eqs. (46) and (47), together with the constraint $\alpha_{1}+\alpha_{2}=1$ and the phasic EOS relations $\mathcal{E}_{k}=\mathcal{E}_{k}\left(p_{k}, \rho_{k}\right)$, give an algebraic system for the equilibrium variables $\alpha_{1}^{\circledast}, p^{\circledast}, T^{\circledast}, \rho_{1}^{\circledast}, \rho_{2}^{\circledast}$. The system can be easily reduced to the solution of a single equation for one variable, for instance the equilibrium pressure $p^{\circledast}$. In general this equation is non-linear and needs to be solved numerically, for example by Newton's iterative method. See Appendix C.3.

Note that with the solution procedure described here we do not need to specify expressions for the interface density $\rho_{\mathrm{I}}$ and the interface specific total energy $e_{\mathrm{I}}$ in the ODEs system (44). Let us also mention that an analogous procedure has also been used in [23].

To end this section, it is important to stress that, similar to the pressure relaxation step described in Section 3.3, these steps of the algorithm for thermal and thermo-chemical equilibrium ensure consistency of the equilibrium pressure values $p^{* *}$ and $p^{\circledast}$ with the correct mixture equation of state. Again, this comes from the fact that by construction the relaxed thermodynamic state satisfies the mixture energy equation (4), and that the discrete values of the mixture total energy $E^{\circledast}=E^{* *}=E^{*}=E^{0}$ computed through our Godunov-type scheme for the solution of the homogeneous system are conservation-consistent.

\section{Numerical experiments}

We present here a selection of numerical results in one and two space dimensions obtained by using the method described in the previous sections with and without activation of thermo-chemical relaxation processes. For the onedimensional tests we also illustrate some comparison results with an alternative algorithm for heat and mass transfer that consists in skipping Step 3a in the procedure described in Section 4.

\subsection{Water cavitation tube problem}

We begin by considering a water liquid-vapor expansion (cavitation) tube problem in one dimension proposed by Saurel et al. [8]. In this test we have a shock tube of unit length filled with liquid water of density $\rho_{l}=1150 \mathrm{~kg} / \mathrm{m}^{3}$ at atmospheric pressure $p=10^{5} \mathrm{~Pa}$. The liquid is assumed to contain a uniformly distributed small amount of vapor, $\alpha_{v}=10^{-2}$ 

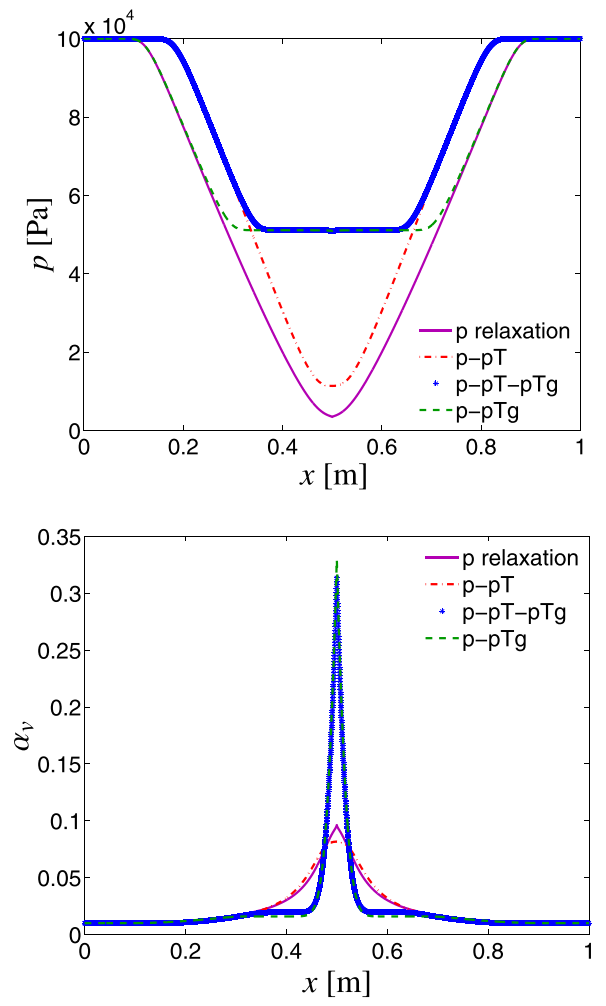
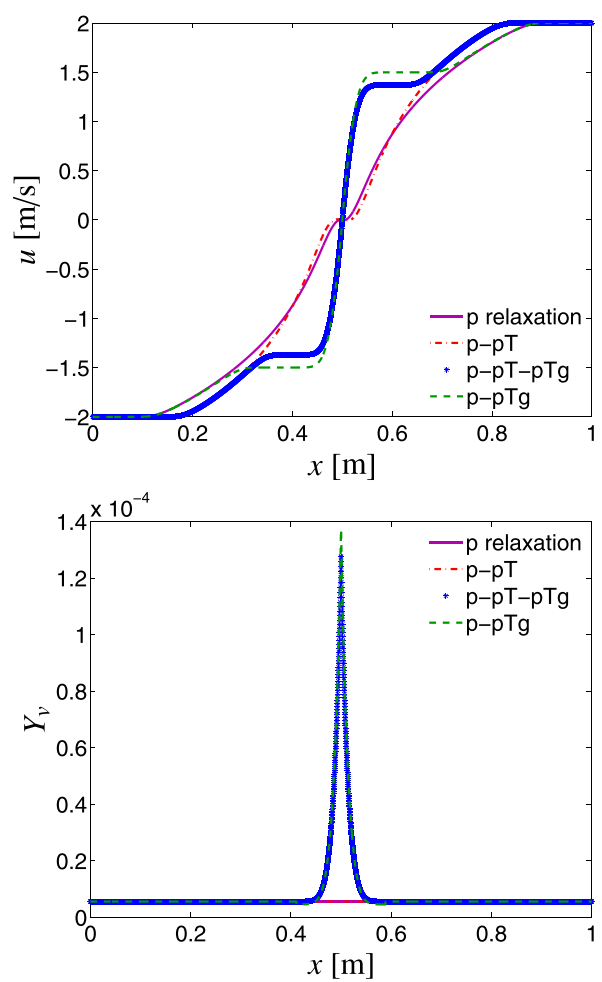

Fig. 1. Numerical results for the water cavitation tube test with initial velocity $|u|=2 \mathrm{~m} / \mathrm{s}$ at time $t=3.2 \mathrm{~ms}$.

Table 1

Parameters for the SG EOS for liquid and vapor water.

\begin{tabular}{llllr}
\hline Phase & $\gamma$ & $\pi[\mathrm{Pa}]$ & $\eta[\mathrm{J} / \mathrm{kg}]$ & $\eta^{\prime}[\mathrm{J} /(\mathrm{kg} \mathrm{K})]$ \\
\hline liquid & 2.35 & $10^{9}$ & $-1167 \times 10^{3}$ & 0 \\
vapor & 1.43 & 0 & $2030 \times 10^{3}$ & 1816 \\
\hline
\end{tabular}

in the whole domain. We use the stiffened gas equation of state (3) to model the thermodynamic behavior of the liquid and vapor phases of water, with the parameters given in Table 1. With this set of data, from (3) we find the liquid temperature as $T_{l}=354.728 \mathrm{~K}$, and hence the vapor density $\rho_{v}=0.63 \mathrm{~kg} / \mathrm{m}^{3}$, by assuming the flow in thermal equilibrium $T_{v}=T_{l}$.

A velocity discontinuity is set at $x=0.5 \mathrm{~m}$ at initial time. In the first test case we set $u=-2 \mathrm{~m} / \mathrm{s}$ on the left and $u=2 \mathrm{~m} / \mathrm{s}$ on the right of the discontinuity.

For this specific test, we perform the computation with four different levels of relaxation by using the basic techniques detailed in Section 4: only mechanical relaxation with no heat and mass transfer (case denoted as $p$ relaxation in the following and in the legend of the figures); mechanical relaxation and thermal relaxation at interfaces ( $p T$ relaxation); mechanical relaxation, thermal relaxation at interfaces and full thermodynamic relaxation at interfaces under metastable conditions ( $p-p T-p T g$ relaxation); mechanical relaxation and full thermodynamic relaxation at interfaces under metastable conditions ( $p-p T g$ relaxation). Phase transition is hence activated only for the two cases of $p-p T-p T g$ and $p-p T g$ relaxation. The former case $(p-p T-p T g)$ corresponds to the physical model of thermodynamic transfer defined in (10) and to the numerical algorithm illustrated in Sections 3 and 4. The latter case $(p-p T g)$ corresponds to an alternative thermodynamic model where we consider $\theta, v=\infty$ if simultaneously $\epsilon_{\mathrm{I}} \leqslant \alpha_{1} \leqslant 1-\epsilon_{\mathrm{I}}$ and $T_{k}>T_{\text {sat }}\left(p_{\text {eq }}\right)$ for either $k=1$ or $k=2$ (see $[8,22]$ ). Numerically, for this case we pass from Step 2 of the fractional step algorithm (pressure equilibrium) to Step $3 \mathrm{~b}$ (full thermodynamic equilibrium).

We use 5000 grid cells, as in [17], and we set the Courant number $\varkappa=0.5$. Note that the authors in [17] need to take $x \approx 0.15$ in order to obtain a stable solution for this problem. Results are displayed in Fig. 1 , where we indicate with a solid line the $p$ relaxation case, with a dotted/dashed line the $p-p T$ case, with a dashed line the $p-p T g$ case, and with stars the $p-p T-p T g$ case. In all the cases we observe two rarefactions propagating in opposite directions that produce a pressure decrease in the middle region of the tube. Note that the leading edge of the two rarefaction waves in the cases of $p-p T$ and $p-p T-p T g$ relaxation moves at a lower speed with respect to the $p$ and $p-p T g$ relaxation cases. This is a consequence of the activation of thermal transfer at interfaces, see (10), which causes expansion to occur under thermal equilibrium. Note however that the flow in the region of the two rarefaction waves is not under chemical equilibrium since here the metastability condition is not met and therefore Gibbs free energy relaxation is not activated. Let us also notice that the lower characteristic speed predicted by our results in conditions of simultaneous pressure and temperature equilibrium with 

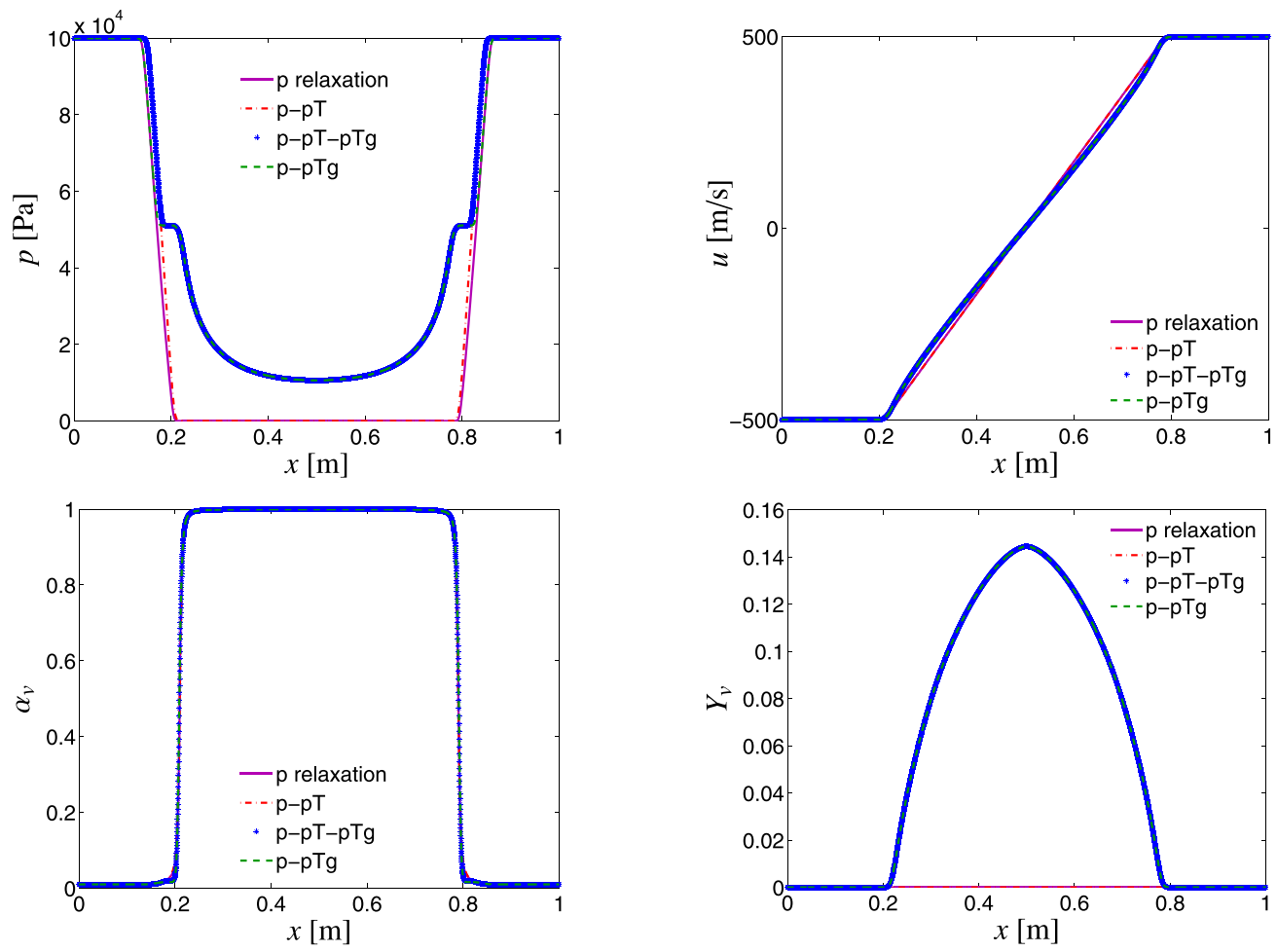

Fig. 2. Numerical results for the water cavitation tube test with initial velocity $|u|=500 \mathrm{~m} / \mathrm{s}$ at time $t=0.58 \mathrm{~ms}$.

respect to the case of merely pressure equilibrium agrees with the theoretical work of Flåtten-Lund [33], where the authors derive analytical expressions of the characteristic equilibrium speeds for a hierarchy of relaxation two-phase models. The wave structure solution with no phase transition is commonly seen in one of the classical mechanical cavitation tube test (cf. [9] and one example obtained with the present method in [51]). When thermal and chemical processes are activated at metastable states (cases $p-p T-p T g$ and $p-p T g$ ) we observe in the solution two additional waves propagating in opposite directions at a speed lower than the speed of the two external expansion waves. These additional waves are evaporation fronts. In these cases with liquid-vapor transition liquid water is expanded until the saturation pressure is reached (see the plot of the pressure in Fig. 1). We can notice from the plot of the vapor mass fraction that a small amount of vapor is generated.

Next, we perform an analogous test but with stronger rarefaction effects by setting the initial velocity to $u=-500 \mathrm{~m} / \mathrm{s}$ on the left of the initial discontinuity and $u=500 \mathrm{~m} / \mathrm{s}$ on the right. We use 5000 grid cells and $\varkappa=0.1$. Due to the stiffness of the problem, taking a higher Courant number deteriorates the accuracy of the pressure solution in correspondence of the evaporation fronts. Results are displayed in Fig. 2, where again we show the four cases corresponding to different levels of relaxation. In this test with stronger expansion the two evaporation waves (one left-going, the other right-going) are more clearly distinguishable, and the increase of the vapor mass fraction is much more significant.

These numerical results agree qualitatively with those in the literature [8,17]. Moreover, our numerical method improves significantly the computational time with respect to the approach of Zein et al. [17]. Here for instance the authors need to take the Courant number as low as $\varkappa=0.03$ for the expansion tube test with strong rarefactions.

\subsection{Dodecane liquid-vapor shock tube problem}

We consider here a dodecane liquid-vapor shock tube problem also proposed in [8]. This test involves a unit length shock tube with an initial discontinuity located at $x=0.75 \mathrm{~m}$ that separates a left region filled with liquid dodecane and a right region filled with vapor dodecane. As in [8], for numerical reasons each fluid region contains a small amount of the phase that fills the region on other side of the discontinuity $\left(\epsilon=10^{-8}\right)$.

The initial condition consists of two constant states on the two sides of the discontinuity with data

$$
\left(\rho_{v}, \rho_{l}, u, p, \alpha_{v}\right)_{L}=\left(2 \mathrm{~kg} / \mathrm{m}^{3}, 500 \mathrm{~kg} / \mathrm{m}^{3}, 0,10^{8} \mathrm{~Pa}, 10^{-8}\right)
$$

and

$$
\left(\rho_{v}, \rho_{l}, u, p, \alpha_{v}\right)_{R}=\left(2 \mathrm{~kg} / \mathrm{m}^{3}, 500 \mathrm{~kg} / \mathrm{m}^{3}, 0,10^{5} \mathrm{~Pa}, 1-10^{-8}\right),
$$

where the subscripts $L$ and $R$ denote the states on the left and on the right of the interface. The liquid and vapor phases of dodecane are modeled through the stiffened gas equation of state (3) with the parameters given in Table 2. 

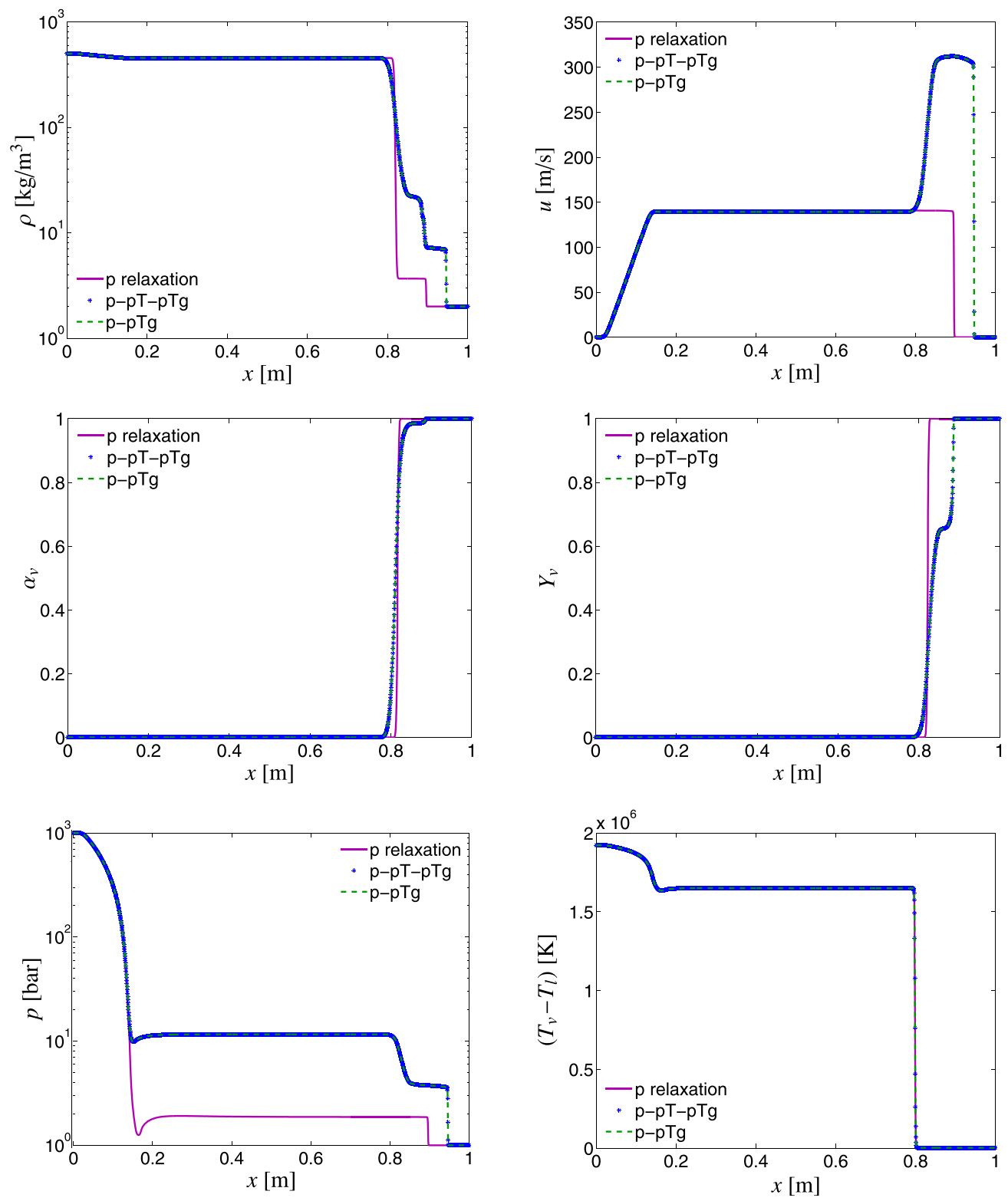

Fig. 3. Numerical results for the dodecane liquid-vapor shock tube problem at time $t=473 \mu$ s. The plots show the solution computed with and without activation of thermo-chemical transfer.

Table 2

Parameters for the SG EOS for liquid and vapor dodecane.

\begin{tabular}{llllc}
\hline Phase & $\gamma$ & $\pi[\mathrm{Pa}]$ & $\eta[\mathrm{J} / \mathrm{kg}]$ & $\eta_{v}[\mathrm{~J} /(\mathrm{kg} \mathrm{K})]$ \\
\hline liquid & 2.35 & $4 \times 10^{8}$ & $-775.269 \times 10^{3}$ & 0 \\
vapor & 1.025 & 0 & $-237.547 \times 10^{3}$ & 1077.7 \\
\hline
\end{tabular}

Fig. 3 shows numerical results at time $t=473 \mu$ s obtained by employing our numerical model with and without mass and heat transfer effects. Here we plot results for three different levels of relaxation: $p$ relaxation (solid line), $p-p T-p T g$ relaxation (stars), and $p-p T g$ relaxation (dashed line). Thermo-chemical transfer at metastable states and therefore liquidvapor transition is activated only in the last two cases. From the plots we can observe for both cases with and without phase transition that the breaking of the initial liquid-vapor interface results in a leftward going rarefaction wave, a rightward going contact discontinuity, and a shock wave. Moreover, when thermal and chemical effects are activated, liquid-vapor phase change occurs, generating an additional evaporation wave between the rarefaction wave and the contact discontinuity. This evaporation front produces a liquid-vapor saturation region at higher speed. For this test there are not visible differences between the $p-p T-p T g$ and $p-p T g$ cases. In particular, note that the left-going rarefaction occurs in a region of almost pure liquid and hence only mechanical relaxation is activated in this zone for all cases. We can observe that indeed in this zone the flow is away from thermal equilibrium by looking at the plot of the temperature difference $T_{v}-T_{l}$ in Fig. 3 . 
Gas volume fraction
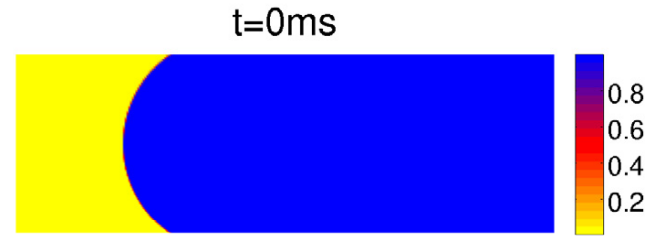

$\mathrm{t}=2 \mathrm{~ms}$

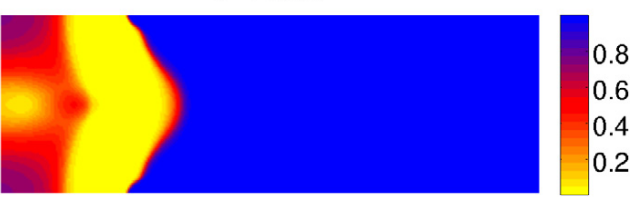

$\mathrm{t}=3.1 \mathrm{~ms}$
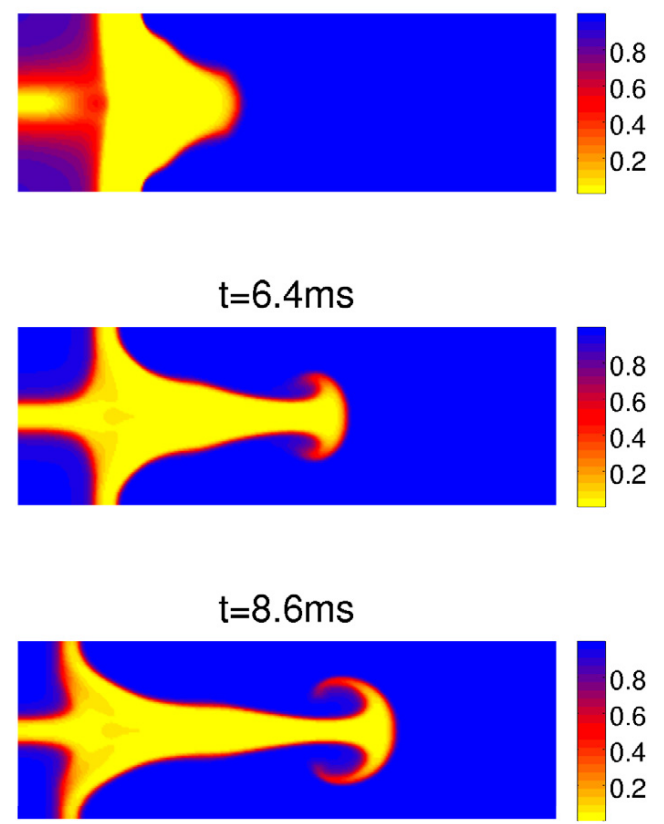

Mixture pressure
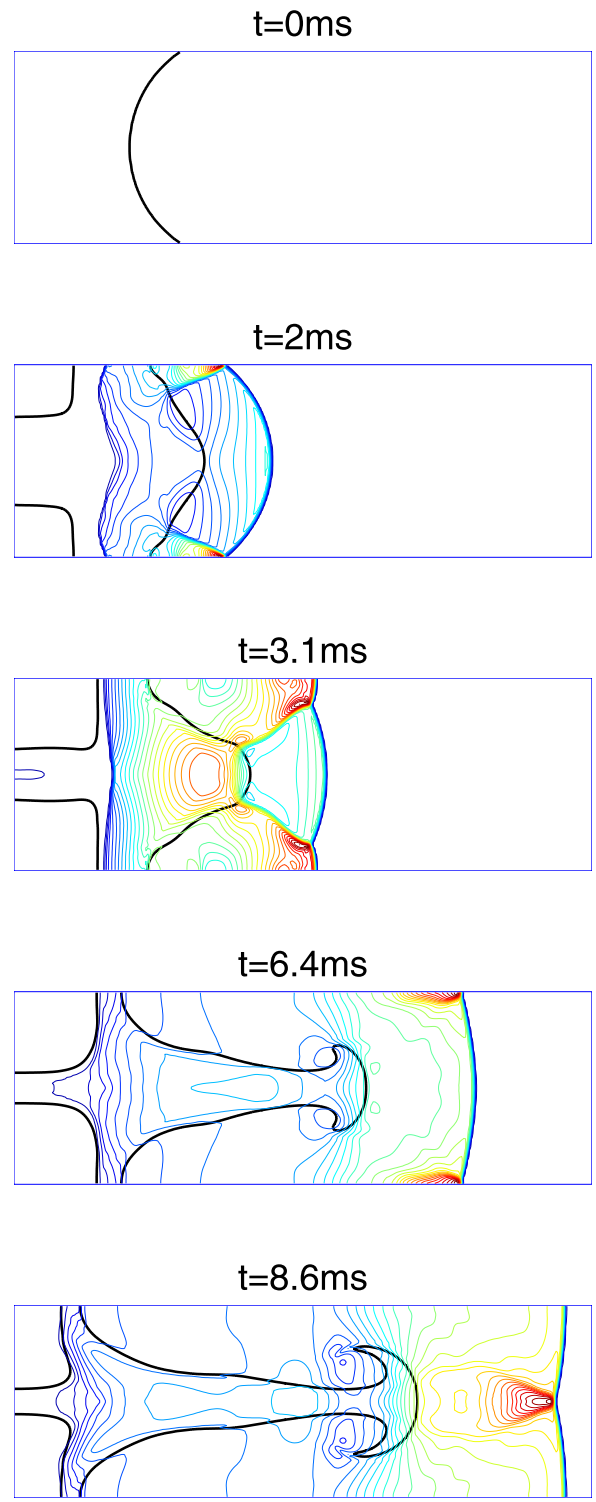

Fig. 4. Numerical results for the cavitating Richtmyer-Meshkov instability experiment. Pseudo-color plots of the gas volume fraction, and contours of the mixture pressure are shown at five different times, $t=0,2,3.1,6.4$ and 8.6 ms using a $300 \times 100$ grid. The black solid line displayed in the pressure plots indicates the approximate location of the gas-water interface.

Our results agree qualitatively with those reported in [8] and [17]. In the present test the computation was performed by using a grid with 2000 cells.

\subsection{Cavitating Richtmyer-Meshkov instability}

Our first example in two space dimensions is the gas-water cavitating Richtmyer-Meshkov instability test problem proposed in [9]. Mass and heat transfer effects are neglected in this experiment. We consider a rectangular region, $(x, y) \in[0,3 \mathrm{~m}] \times[0,1 \mathrm{~m}]$, where at initial time a curved interface separates a left zone filled with nearly pure water $\left(\alpha_{g}=10^{-6}\right)$ and a right zone filled with nearly pure gas $\left(\alpha_{w}=10^{-6}\right)$, see the top row of Fig. 4 for an illustration. The initial pressure is $p=10^{5} \mathrm{~Pa}$ and the densities are $\rho_{g}=100 \mathrm{~kg} / \mathrm{m}^{3}$ and $\rho_{w}=1000 \mathrm{~kg} / \mathrm{m}^{3}$ for gas and water, respectively. Both gas and water have an initial velocity of $u=-200 \mathrm{~m} / \mathrm{s}$. The two materials are modeled by the stiffened gas equation of state with parameters $\gamma_{w}=4.4, \pi_{w}=6 \times 10^{8} \mathrm{~Pa}, \eta_{w}=\eta_{w}^{\prime}=0 \mathrm{~Pa}$, for water, and $\gamma_{g}=1.4, \pi_{g}=0 \mathrm{~Pa}, \eta_{g}=\eta_{g}^{\prime}=0 \mathrm{~Pa}$, for the gas. Here, top, bottom and left boundaries are treated as solid walls, whereas the right side is considered an outflow boundary.

Fig. 4 shows the numerical results obtained by using our method at five different times, $t=2,3.1,6.4$ and 8.6 ms, employing a $300 \times 100$ grid. From the plots one can observe that when the flow impinges against the left wall a right-going shock wave propagates through the curved gas-water interface. This produces a Richtmyer-Meshkov instability characterized 
by expansion waves and an elongating jet. The pressure decrease in the expansion zones close to the left wall generates cavitation pockets in this region. Our results agree well with the ones shown in [9], by looking at the global features of the solution structure.

\subsection{Vapor bubble compression}

We are next interested in simulating the piston-induced compression of a vapor bubble in a liquid and the associated phase transition processes. We adopt a problem setup similar to the one considered by Caro et al. in [53] (see also [14]). Initially a stationary vapor bubble of radius $0.2 \mathrm{~m}$ is situated at the center of a liquid-filled unit square container with a wall-mounted piston on the left boundary and fixed solid walls on the other sides. We fix phasic pressures and temperatures to the equilibrium values $p_{l}=p_{v}=10^{5} \mathrm{~Pa}$, and $T_{l}=T_{v}=600 \mathrm{~K}$, respectively. We assume that the material inside the container is dodecane, and the parameters for the liquid and vapor phases are taken as in Table 2. With this set of data, the phasic densities for the liquid and vapor phases are then $\rho_{l}=458.338 \mathrm{~kg} / \mathrm{m}^{3}$ and $\rho_{v}=3.408 \mathrm{~kg} / \mathrm{m}^{3}$. Similar to previous experiments, initially each fluid is assumed to be nearly pure, with $\alpha_{l}=10^{-6}$ in the vapor bubble, and $\alpha_{v}=10^{-6}$ in the surrounding liquid region, respectively. The piston velocity set at the left wall is $u_{p}=100 \mathrm{~m} / \mathrm{s}$. See [19] for more details on the numerical treatment for this moving boundary.

Numerical results obtained with the full model with thermo-chemical transfer are shown in Fig. 5. Here we display pseudo-color plots for the vapor mass fraction and contour plots for the mixture pressure at three different times, $t=0.4$, 0.8 , and $1.2 \mathrm{~ms}$, using a $200 \times 200$ grid. Due to the piston motion and consequent bubble compression, the initial circular shape of the vapor bubble is deformed to a kidney shape, as it is clearly visible from the vapor mass fraction plots. Let us note that no spurious pressure oscillations at interfaces are observed at the various stages of the bubble compression, this indicating the efficiency of both the wave propagation scheme and the thermodynamic relaxation procedure described in Section 4.

\subsection{High-velocity underwater projectile}

To show the capabilities of our two-dimensional computational method with mapped grids for irregularly-shaped domains, we begin by considering the high-velocity underwater projectile numerical experiment presented in [8] (see also [10]). In this problem liquid water flows at speed $u=600 \mathrm{~m} / \mathrm{s}$ from left to right over an immersed obstacle with hexagonal section. Initially the liquid is at atmospheric pressure, $p=10^{5} \mathrm{~Pa}$, and it has density $\rho_{l}=1500 \mathrm{~kg} / \mathrm{m}^{3}$. A small amount of vapor $\alpha_{v}=10^{-3}$ is present in the liquid at the initial time. The parameters of the SG equation of state for the liquid and vapor phases of water are the same as those used for the one-dimensional cavitation tube experiment in Section 5.1, see Table 1.

Due to the symmetry of the problem, we perform the computation only on the portion of the physical domain above the symmetry axis, set at $y=0$, imposing a line-of-symmetry boundary condition at this axis. We use a uniform grid with $600 \times 200$ cells over the rectangular computational domain $[0,1.5 \mathrm{~m}] \times[0,0.5 \mathrm{~m}]$, which is mapped to a curvilinear grid in the physical domain, see Fig. 6.

We compute the solution with and without heat and mass transfer. In both cases the flow interaction with the edges of the obstacle generates strong rarefaction waves, which determine a pressure decrease and consequently a cavitation region. After a time sufficiently large the flow reaches a steady configuration with a stationary cavitation zone. In the case with no thermo-chemical effects only mechanical cavitation is observed, that is the growth of a gas pocket due to the pressure decrease. Fig. 7 shows steady-state results at time $t=10 \mathrm{~ms}$. Here we display pseudo-color plots of the vapor volume fraction, vapor mass fraction, mixture density, and mixture pressure obtained by our method with and without activation of the thermo-chemical solver. By looking at the results with heat and mass transfer in the left column of the figure, one can observe the variation of the vapor mass fraction, which indicates the formation of evaporation fronts. The phasic mass fractions remain instead constant if no phase transition is modeled, and in this case only the volume fractions vary, as shown in the results in the right column of the figure. All these results are in agreement with those presented in [8]. Moreover, as in [8], we observe a different pressure field in correspondence of the cavitation wake: while for the case with no thermo-chemical effects the pressure continues decreasing reaching small values of the order of $10^{-3}$ bar, for the case with thermo-chemical transfer the pressure decreases until saturation conditions, corresponding approximately to a value of 0.5 bar. This is analogous to the behavior of the pressure observed for the one-dimensional cavitation test in Section 5.1 (Fig. 1).

\subsection{High-pressure fuel injector}

To end this section, we perform the high-pressure fuel injector experiment proposed in [8]. Here we consider a nozzle where liquid fuel (dodecane) is injected from a high-pressure tank to a chamber at atmospheric pressure. The nozzle has the shape shown in Fig. 6, and it has a length of $10 \mathrm{~cm}$ and a height of $4 \mathrm{~cm}$. The height of the throat is $1.2 \mathrm{~cm}$, and the outer inclination angles of the converging and diverging chambers with respect to the horizontal direction are $45^{\circ}$ and $10^{\circ}$, respectively. We set an initial discontinuity at $\bar{x}=0.8 \mathrm{~cm}$ between a region of liquid dodecane at a pressure of 1000 bar and at a temperature of $640 \mathrm{~K}$ (the corresponding density is $570 \mathrm{~kg} / \mathrm{m}^{3}$ ) and a region of dodecane vapor at atmospheric 
Vapor mass fraction

\section{$\mathrm{t}=0.4 \mathrm{~ms}$}

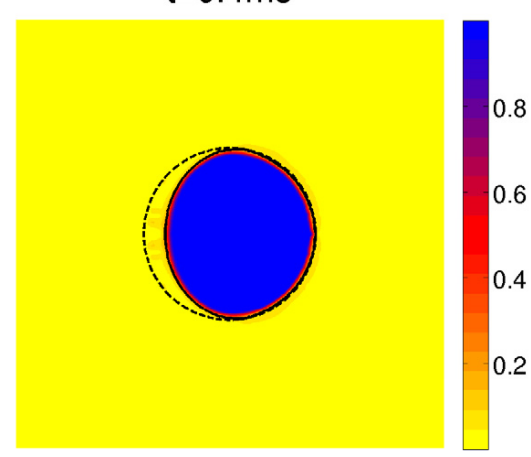

\section{$\mathrm{t}=0.8 \mathrm{~ms}$}

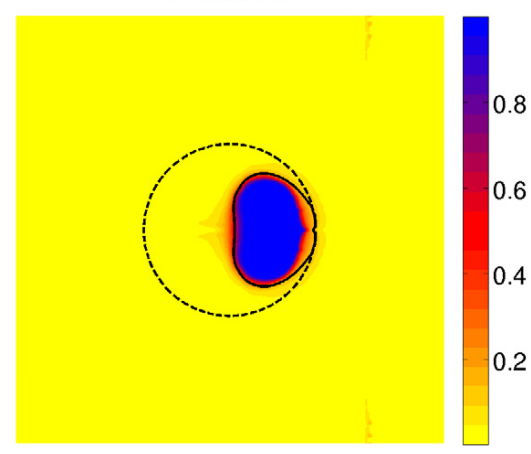

$\mathrm{t}=1.2 \mathrm{~ms}$

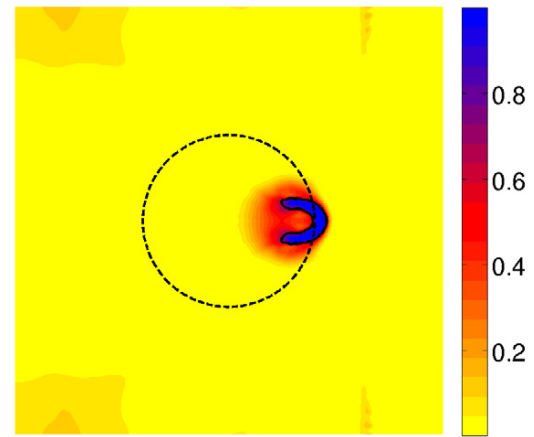

Pressure
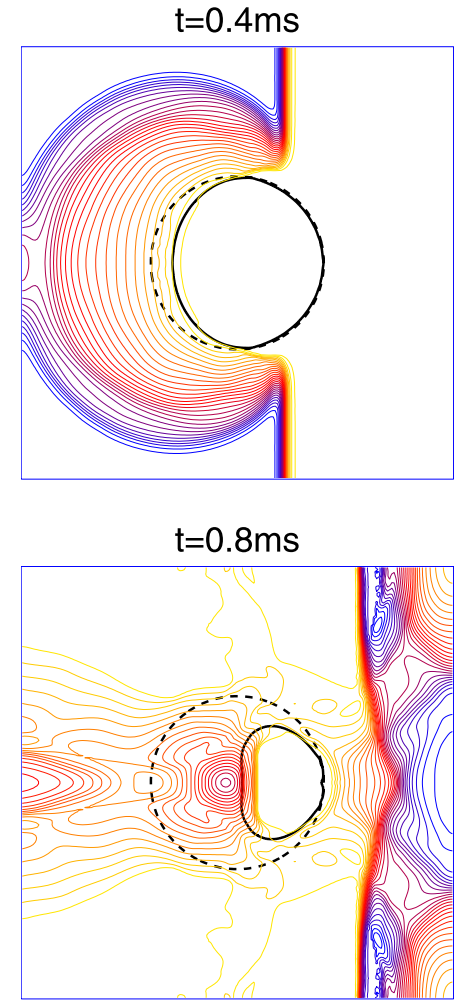

$\mathrm{t}=1.2 \mathrm{~ms}$

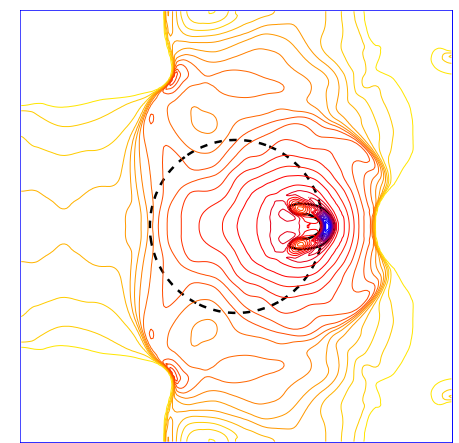

Fig. 5. Numerical results for the vapor-bubble compression test with thermo-chemical relaxation effects. Pseudo-color plots of the vapor mass fraction (left) and contour plots of the pressure (right) are shown at three different times, $t=0.4,0.8$, and $1.2 \mathrm{~ms}$, using a $200 \times 200$ grid. In each plot, the dashed line indicates the initial location of the liquid-vapor interface, and the solid line indicates the location of the interface at the displayed time.

Grid for the projectile experiment

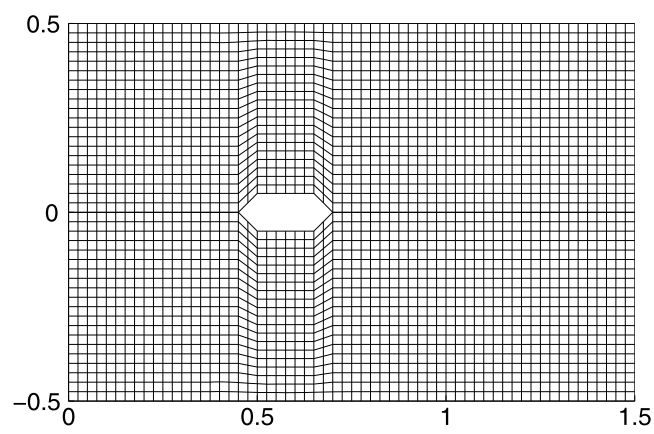

Grid for the injector experiment

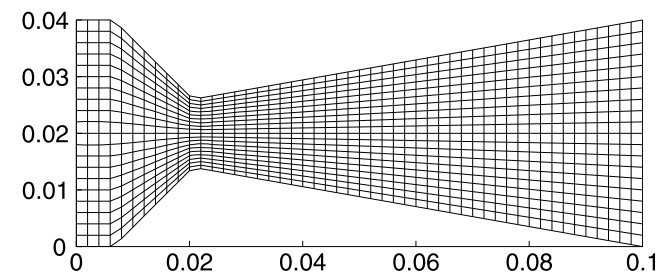

Fig. 6. Types of quadrilateral grid used for the high-speed underwater projectile experiment (on the left), and for the high-pressure injector experiment (on the right). 
With thermo-chemical relaxation
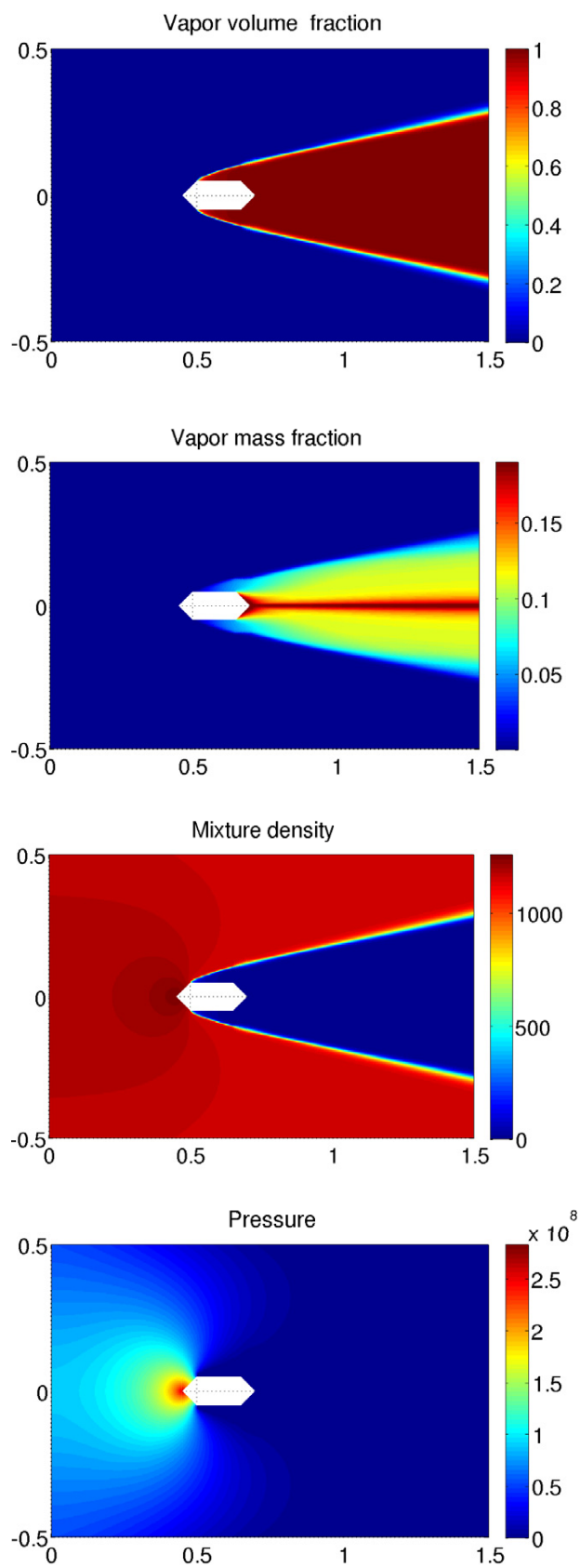

No thermo-chemical relaxation
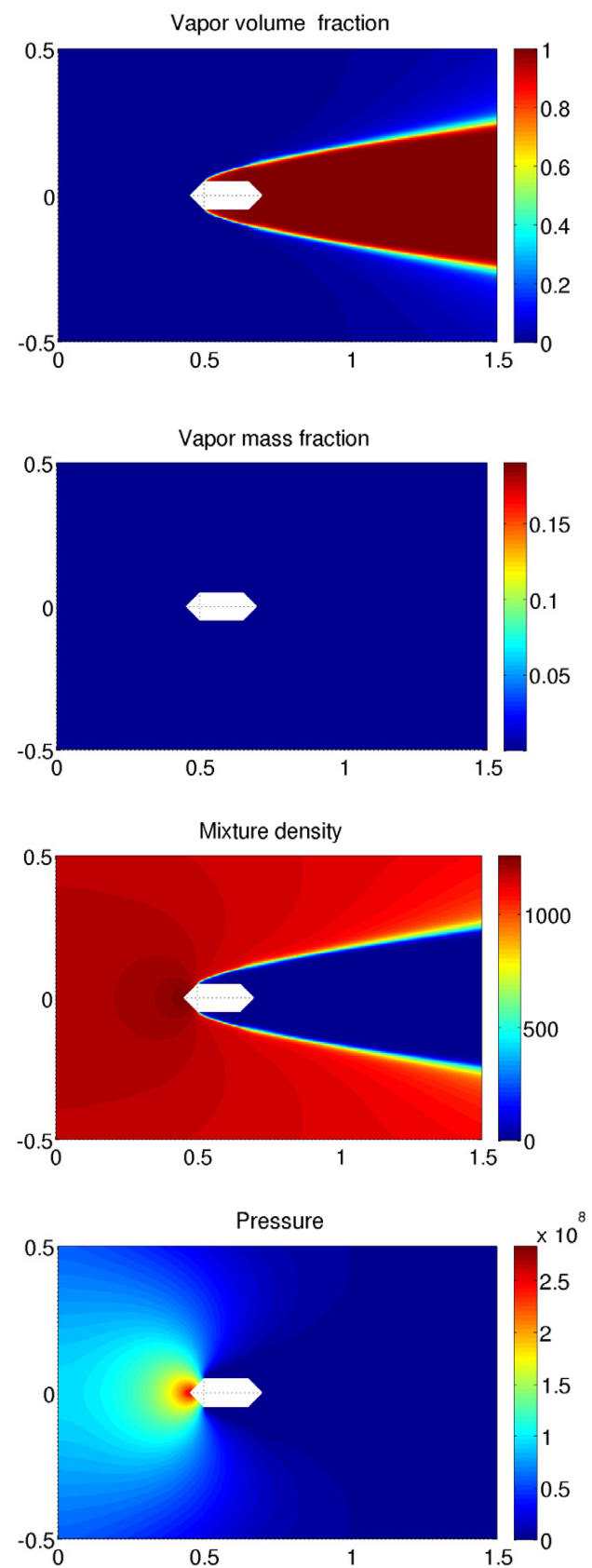

Fig. 7. Steady state results for the high-speed underwater projectile experiment. Pseudo-color plots of the vapor volume fraction, vapor mass fraction, mixture density, and mixture pressure obtained with (left column) and without (right column) activation of the thermo-chemical solver.

conditions (1 bar). A small amount of vapor $\alpha_{v}=10^{-4}$ is present in the liquid at the initial time. The parameters of the SG equation of state for the liquid and vapor dodecane phases are the same as those used in the previous one-dimensional dodecane liquid-vapor shock tube problem, see Table 2.

Numerical results obtained by our method with and without heat and mass transfer over a $400 \times 160$ grid are shown in Fig. 8. Here we display pseudo-color plots of the vapor volume fraction, vapor mass fraction, mixture density, vapor temperature and mixture pressure at time $t=600 \mu \mathrm{s}$, corresponding to a fully-developed stage on the fuel jet. As observed in [8], there are not large differences in the size of the cavitation pockets between the case with phase transition (left column) and the case without phase transition (right column), as one can notice from the plots of the volume fraction and of the density. Significant differences can be nonetheless observed in the vapor mass fraction and vapor temperature plots. Let us note in particular the sharp variation of the vapor temperature across the interfacial zones if thermo-chemical effects are not activated. 
With thermo-chemical relaxation

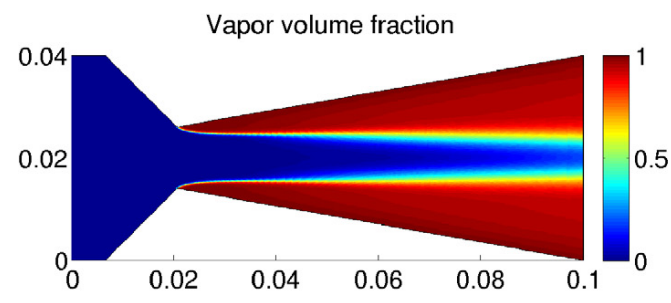

Vapor mass fraction

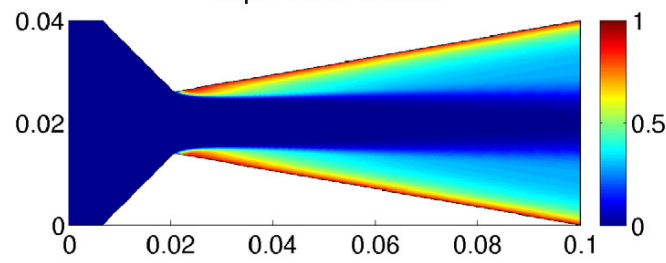

Mixture density

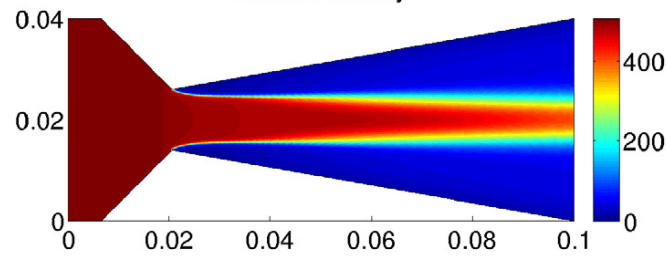

Vapor temperature
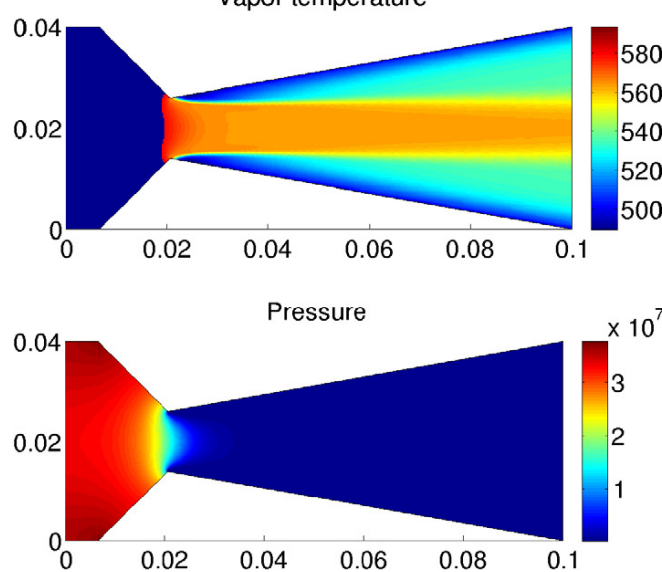

No thermo-chemical relaxation

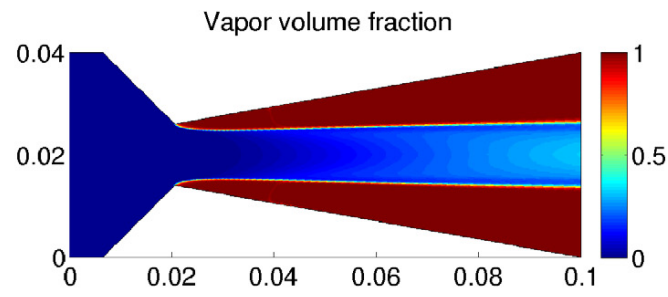

Vapor mass fraction

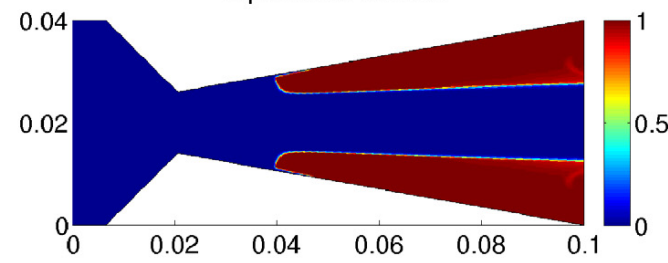

Mixture density

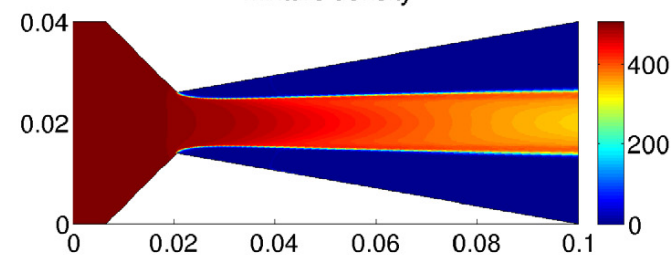

Vapor temperature
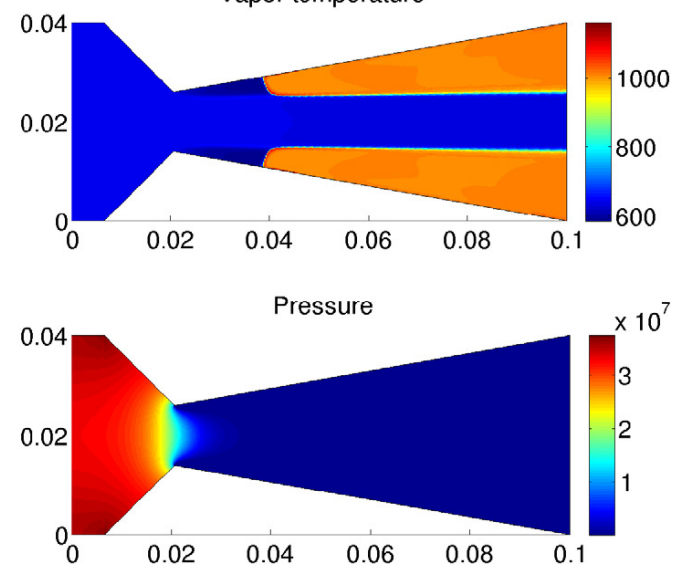

Fig. 8. Numerical results for the high-pressure fuel injector experiment. Pseudo-color plots of the vapor volume fraction, vapor mass fraction, mixture density, vapor temperature, and mixture pressure are shown at time $t=600 \mu$ s using a $400 \times 160$ grid. The results with heat and mass transfer are displayed in the left column, while the results without heat and mass transfer are displayed in the right column.

\section{Conclusions}

We have developed a new numerical model for two-phase compressible flows with cavitation and liquid-vapor transition based on the single-velocity 6-equation two-phase flow model with stiff pressure relaxation of Saurel et al. [9]. The model includes thermal and chemical potential relaxation terms to account for heat and mass transfer processes. The key idea of our method is to employ in the numerical discretization a phasic-total-energy-based formulation of the basic hyperbolic model system rather than the phasic-internal-energy-based formulation used previously in the literature [9,17]. Our approach easily allows us to design a numerical scheme that guarantees consistency with conservation of the mixture total energy with no need of augmenting the 6-equation system with an extra conservation law for the mixture energy, as instead done for the classical model [9,17]. A fully-discretized two-dimensional high-resolution scheme has been developed for the model equations, which employs a wave propagation method for the approximation of the homogeneous hyperbolic portion of the system. Thermo-chemical source terms are handled through efficient stiff relaxation solvers that drive the two-phase mixture to thermodynamic equilibrium conditions via the solution of simple algebraic systems of equations. Thanks to the conservation consistency property of the method, these numerical relaxation procedures ensure also consistency of the relaxed pressure at equilibrium with the correct mixture equation state. Numerical experiments in one 
and two space dimensions show the ability of the proposed numerical model to simulate cavitation pockets dynamics and liquid-vapor transition processes.

Although the six-equation numerical model of Saurel et al. [9] with stiff pressure relaxation has proven to be very effective, our phasic-total-energy-based approach provides a simpler scheme. Moreover, we think that the mixture-energyconsistency property of our method is a key factor for the efficiency and robustness of the sequence of relaxation steps for mechanical and thermo-chemical equilibrium that follows the solution of the homogeneous 6-equation system. In particular, at least for some of the numerical tests with cavitation that we have reported, our method improves remarkably the computational time of the 6-equation two-phase model with liquid-vapor transition of Zein et al. [17], proving to be stable for higher Courant numbers. Let us mention that only one-dimensional numerical experiments were presented in [17].

Due to its simplicity and robustness the proposed numerical model appears to be suited for extensions to related multiphase problems, such as the dynamics of powder compaction [54], and to more complex flow regimes. One challenging topic for future developments is the study of low-Mach numerical strategies for the current two-phase model. Indeed the well known difficulties encountered by compressible flow solvers for low Mach number regimes (cf. [55-58,23]) represent a critical issue for liquid-gas flows with cavitation and evaporation. This is due to the large and rapid variation of the acoustic impedance in the medium, where highly compressible fluid regions (vapor) and nearly incompressible zones (liquid) co-exist and interact.

\section{Acknowledgements}

The first author (M. Pelanti) was partially supported by DGA, the French Government General Directorate for Armament (Direction Générale de l'Armement), in the framework of a research project funded by DGA on "Full-Mach-number-range numerical simulation of cavitating flows by multiphase compressible flow models", Grants DGA N. 2009.60.035.00.470.75.01 and DGA N. 2012.60.0011.00.470.75.01. The second author (K.-M. Shyue) was supported in part by the National Science Council of Taiwan, Grant NSC 99-2115-M-002-005-MY2.

\section{Appendix A. Model system eigenstructure}

For completeness of the illustration of the proposed approximate Riemann solvers, we report here the eigenstructure of the one-dimensional (homogeneous) 6-equation model $\partial_{t} q+\partial_{x} f(q)+\sigma\left(q, \partial_{x} q\right)=0$. The eigenvalues are

$$
\lambda_{1}=u-c, \quad \lambda_{2}=\lambda_{3}=\lambda_{4}=\lambda_{5}=u, \quad \lambda_{6}=u+c,
$$

where $u$ is the velocity in the $x$ direction, and $c$ the mixture sound speed in (2). The matrix $R=\left[r_{1}, \ldots, r_{6}\right]$ of the corresponding right eigenvectors $r_{k}, k=1, \ldots, 6$, can be taken as

$$
R(q)=\left(\begin{array}{cccccc}
0 & 0 & 0 & 0 & 1 & 0 \\
Y_{1} & 0 & 0 & 1 & 0 & Y_{1} \\
Y_{2} & 0 & 1 & 0 & 0 & Y_{2} \\
u-c & 0 & u & u & 0 & u+c \\
Y_{1}\left(H_{1}-u c\right) & -\frac{\kappa_{2}}{\kappa_{1}} & \frac{\kappa_{2}}{\kappa_{1}} H_{2}-\frac{c_{2}^{2}}{\kappa_{1}} & H_{1}-\frac{c_{1}^{2}}{\kappa_{1}} & \frac{\Pi_{1}-\Pi_{2}}{\kappa_{1}} & Y_{1}\left(H_{1}+u c\right) \\
Y_{2}\left(H_{2}-u c\right) & 1 & 0 & 0 & 0 & Y_{2}\left(H_{2}+u c\right)
\end{array}\right)
$$

where we have denoted with $H_{k}=h_{k}+\frac{u^{2}}{2}$ the specific total enthalpy of phase $k$, and where we have defined $\Pi_{k}=-\rho_{k} c_{k}^{2}+$ $p_{k}\left(1+\kappa_{k}\right)$, with $\kappa_{k}=\frac{\partial p_{k}\left(\mathcal{E}_{k}, \rho_{k}\right)}{\partial \mathcal{E}_{k}}, k=1,2$.

\section{Appendix B. Roe matrix and eigenstructure}

By imposing the conservation conditions (31) a Roe matrix for the 6-equation model closed with the SG EOS can be determined as $\tilde{A}=A\left(\hat{u}, \hat{Y}_{1}, \widetilde{u Y_{1}}, \widehat{Y_{1} H_{1}}, \widehat{Y_{2} H_{2}}\right)$,

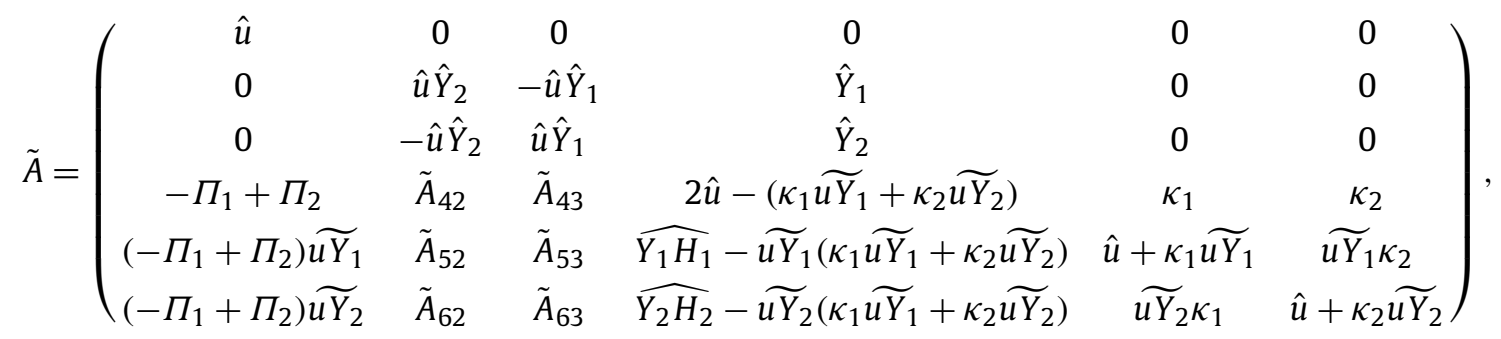

where here (SG EOS) $\Pi_{k}=\gamma_{k} \pi_{k}$, and where 


$$
\begin{aligned}
& \tilde{A}_{42}=-\hat{u}^{2}+\chi_{1}-\kappa_{1} \frac{\hat{u}^{2}}{2}+\hat{u}\left(\kappa_{1} \widetilde{u Y_{1}}+\kappa_{2} \widetilde{u Y}_{2}\right), \\
& \tilde{A}_{43}=-\hat{u}^{2}+\chi_{2}-\kappa_{2} \frac{\hat{u}^{2}}{2}+\hat{u}\left(\kappa_{1} \widetilde{u Y_{1}}+\kappa_{2} \widetilde{u Y}_{2}\right) \text {, } \\
& \tilde{A}_{52}=-\widehat{Y_{1} H_{1}} \hat{u}+\widetilde{u Y_{1}}\left(\chi_{1}-\kappa_{1} \frac{\hat{u}^{2}}{2}\right)+\hat{u} u \widetilde{Y}_{1}\left(\kappa_{1} \widetilde{u Y_{1}}+\kappa_{2} \widetilde{u Y_{2}}\right) \text {, } \\
& \tilde{A}_{53}=-\widehat{Y_{1} H_{1}} \hat{u}+\widetilde{u Y_{1}}\left(\chi_{2}-\kappa_{2} \frac{\hat{u}^{2}}{2}\right)+\hat{u} u \widetilde{Y}_{1}\left(\kappa_{1} \widetilde{u Y_{1}}+\kappa_{2} \widetilde{u Y_{2}}\right) \text {, } \\
& \tilde{A}_{62}=-\widehat{Y_{2} H_{2}} \hat{u}+\widetilde{u Y_{2}}\left(\chi_{1}-\kappa_{1} \frac{\hat{u}^{2}}{2}\right)+\hat{u} \widetilde{u Y_{2}}\left(\kappa_{1} \widetilde{u Y_{1}}+\kappa_{2} \widetilde{u Y_{2}}\right) \text {, } \\
& \tilde{A}_{63}=-\widehat{Y_{2} H_{2}} \hat{u}+\widetilde{u Y_{2}}\left(\chi_{2}-\kappa_{2} \frac{\hat{u}^{2}}{2}\right)+\hat{u} u \widetilde{Y}_{2}\left(\kappa_{1} \widetilde{u Y_{1}}+\kappa_{2} \widetilde{u Y_{2}}\right) \text {. }
\end{aligned}
$$

The averaged quantities $\hat{u}, \hat{Y}_{1}, \hat{Y}_{2}, \widetilde{u Y_{1}}, \widetilde{u Y_{2}}, \widehat{Y_{1} H_{1}}, \widehat{Y_{2} H_{2}}$ in the matrix above are defined as

$$
\begin{aligned}
& \hat{u}=\frac{u_{\ell} \sqrt{\rho_{\ell}}+u_{r} \sqrt{\rho_{r}}}{\sqrt{\rho_{\ell}}+\sqrt{\rho_{r}}}, \\
& \hat{Y}_{k}=\frac{Y_{k \ell} \sqrt{\rho_{\ell}}+Y_{k r} \sqrt{\rho_{r}}}{\sqrt{\rho_{\ell}}+\sqrt{\rho_{r}}}, \quad k=1,2, \\
& \widehat{u Y_{k}}=\frac{\left(u Y_{k}\right)_{\ell} \sqrt{\rho_{\ell}}+\left(u Y_{k}\right)_{r} \sqrt{\rho_{r}}}{\sqrt{\rho_{\ell}}+\sqrt{\rho_{r}}}, \quad k=1,2, \\
& \widetilde{u Y_{k}}=\frac{1}{2}\left(\hat{u} \hat{Y}_{k}+\widehat{u Y_{k}}\right), \quad k=1,2, \\
& \widehat{Y_{k} H_{k}}=\frac{\left(Y_{k} H_{k}\right)_{\ell} \sqrt{\rho_{\ell}}+\left(Y_{k} H_{k}\right)_{r} \sqrt{\rho_{r}}}{\sqrt{\rho_{\ell}}+\sqrt{\rho_{r}}}, \quad k=1,2 .
\end{aligned}
$$

Note that $\hat{Y}_{1}+\hat{Y}_{2}=1$ and $\widetilde{u Y}_{1}+\widetilde{u Y_{2}}=\hat{u}$. Moreover the corresponding average sound speed is

$$
\tilde{c}=\sqrt{\widetilde{Y_{1} c_{1}^{2}}+\widetilde{Y_{2} c_{2}^{2}}}
$$

where $\widetilde{Y_{k} c_{k}^{2}}=\kappa_{k}\left(\widehat{Y_{k} H_{k}}-\frac{\hat{u}^{2}}{2} \hat{Y}_{k}\right)+\chi_{k} \hat{Y}_{k}$.

The Roe eigenvalues are given by

$$
\tilde{\lambda}_{1}=\hat{u}-\tilde{c}, \quad \tilde{\lambda}_{2}=\tilde{\lambda}_{3}=\tilde{\lambda}_{4}=\tilde{\lambda}_{5}=\hat{u}, \quad \tilde{\lambda}_{6}=\hat{u}+\tilde{c},
$$

and the corresponding matrix of the Roe right eigenvectors, $\tilde{R}=\left[\tilde{r}_{1}, \ldots, \tilde{r}_{6}\right]$, is

$$
\tilde{R}=\left(\begin{array}{cccccc}
0 & 0 & 0 & 0 & 1 & 0 \\
\hat{Y}_{1} & 0 & 0 & 1 & 0 & \hat{Y}_{1} \\
\hat{Y}_{2} & 0 & 1 & 0 & 0 & \hat{Y}_{2} \\
\hat{u}-\tilde{c} & 0 & \hat{u} & \hat{u} & 0 & \hat{u}+\tilde{c} \\
\widehat{Y_{1} H_{1}}-\widetilde{u Y_{1}} \tilde{c} & -\frac{\kappa_{2}}{\kappa_{1}} & -\frac{\chi_{2}}{\kappa_{1}}+\frac{\kappa_{2}}{\kappa_{1}} \frac{\hat{u}^{2}}{2} & -\frac{\chi_{1}}{\kappa_{1}}+\frac{\hat{u}^{2}}{2} & \frac{\Pi_{1}-\Pi_{2}}{\kappa_{1}} & \widehat{Y_{1} H_{1}}+\widetilde{u Y_{1}} \tilde{c} \\
\widehat{Y_{2} H_{2}}-\widetilde{u Y_{2}} \tilde{c} & 1 & 0 & 0 & 0 & \widehat{Y_{2} H_{2}}+\widetilde{u Y_{2}} \tilde{c}
\end{array}\right) .
$$

The strengths $\tilde{\beta}_{\xi}, \xi=1, \ldots, 6$ of the Roe waves in (32) are obtained through the eigen-decomposition $q_{r}-q_{\ell}=$ $\sum_{\xi=1}^{6} \tilde{\beta}_{\xi} \tilde{r}_{\xi}$, and they are given by

$$
\begin{aligned}
& \tilde{\beta}_{1,6}=\frac{\Delta\left(\alpha_{1} p_{1}+\alpha_{2} p_{2}\right) \mp \tilde{c} \sqrt{\rho_{\ell} \rho_{r}} \Delta u}{2 \tilde{c}^{2}}, \\
& \tilde{\beta}_{2}=-\frac{\Delta\left(\alpha_{1} p_{1}+\alpha_{2} p_{2}\right)}{\tilde{c}^{2}} \widehat{Y_{2} H_{2}}+\frac{\hat{u}^{2}}{2} \Delta\left(\alpha_{2} \rho_{2}\right)+\Delta\left(\alpha_{2} \mathcal{E}_{2}\right), \\
& \tilde{\beta}_{3}=\Delta\left(\alpha_{2} \rho_{2}\right)-\hat{Y}_{2} \frac{\Delta\left(\alpha_{1} p_{1}+\alpha_{2} p_{2}\right)}{\tilde{c}^{2}}, \\
& \tilde{\beta}_{4}=\Delta\left(\alpha_{1} \rho_{1}\right)-\hat{Y}_{1} \frac{\Delta\left(\alpha_{1} p_{1}+\alpha_{2} p_{2}\right)}{\tilde{c}^{2}}, \\
& \tilde{\beta}_{5}=\Delta \alpha_{1},
\end{aligned}
$$

where $\Delta(\cdot) \equiv(\cdot)_{r}-(\cdot)_{\ell}$. 
The definition of the Roe's matrix reported above is easily extended to the two-dimensional case $(\vec{u}=(u, v))$. In this case we need the Roe eigenstructure of the system for a plane-wave Riemann problem in the $x$ and $y$ directions. We use the same averages as in (B.2), except that now in all the formulas kinetic energy terms must take into account the contribution of the tangential velocity $v$ (e.g. $\frac{u^{2}+v^{2}}{2}$ is used instead of $\frac{u^{2}}{2}$ ). For instance, the Roe eigenstructure $\left\{\tilde{\lambda}_{\xi}^{x}, \tilde{r}_{\xi}^{x}\right\}_{\xi=1, \ldots, 7}$ corresponding to the matrix $A^{x}(q) \in \mathbb{R}^{7 \times 7}$ of the system of the plane-wave Riemann problem in the $x$ direction is found as

$$
\tilde{\lambda}_{1}^{x}=\hat{u}-\tilde{c}, \quad \tilde{\lambda}_{2}^{x}=\tilde{\lambda}_{3}^{x}=\tilde{\lambda}_{4}^{x}=\tilde{\lambda}_{5}^{x}=\tilde{\lambda}_{6}^{x}=\hat{u}, \quad \tilde{\lambda}_{7}^{x}=\hat{u}+\tilde{c}
$$

$$
\tilde{R}^{x}=\left(\begin{array}{ccccccc}
0 & 0 & 0 & 0 & 1 & 0 & 0 \\
\hat{Y}_{1} & 0 & 0 & 1 & 0 & 0 & \hat{Y}_{1} \\
\hat{Y}_{2} & 0 & 1 & 0 & 0 & 0 & \hat{Y}_{2} \\
\hat{u}-\tilde{c} & 0 & \hat{u} & 0 & 0 & 0 & \hat{u}+\tilde{c} \\
\hat{v} & 0 & 0 & 0 & 0 & 1 & \hat{v} \\
\widehat{Y_{1} H_{1}}-\widetilde{u Y_{1}} \tilde{c} & -\frac{\kappa_{2}}{\kappa_{1}} & -\frac{\chi_{2}}{\kappa_{1}}+\frac{\kappa_{2}}{\kappa_{1}} \tilde{\mathcal{K}}-\hat{v} \frac{\tilde{V}}{\kappa_{1}} & -\frac{\chi_{1}}{\kappa_{1}}+\tilde{\mathcal{K}}-\hat{v} \frac{\tilde{V}}{\kappa_{1}} & \frac{\Pi_{1}-\Pi_{2}}{\kappa_{1}} & \frac{\tilde{V}}{\kappa_{1}} & \widehat{Y_{1} H_{1}}+\widetilde{u Y_{1}} \tilde{c} \\
\widehat{Y_{2} H_{2}}-\widetilde{u Y_{2}} \tilde{c} & 1 & 0 & 0 & 0 & 0 & \widehat{Y_{2} H_{2}}+\widetilde{u Y_{2}} \tilde{c}
\end{array}\right),
$$

where

$$
\begin{aligned}
& \hat{v}=\frac{v_{\ell} \sqrt{\rho_{\ell}}+v_{r} \sqrt{\rho_{r}}}{\sqrt{\rho_{\ell}}+\sqrt{\rho_{r}}}, \\
& \widehat{v Y_{k}}=\frac{\left(v Y_{k}\right)_{\ell} \sqrt{\rho_{\ell}}+\left(v Y_{k}\right)_{r} \sqrt{\rho_{r}}}{\sqrt{\rho_{\ell}}+\sqrt{\rho_{r}}}, \quad k=1,2, \\
& {\widetilde{v Y_{k}}}=\frac{1}{2}\left(\hat{v} \hat{Y}_{k}+{\widehat{v Y_{k}}}\right), \quad k=1,2, \\
& \tilde{c}=\sqrt{\widetilde{Y_{1} c_{1}^{2}}+\widetilde{Y_{2} c_{2}^{2}}}, \quad \widetilde{Y_{k} c_{k}^{2}}=\kappa_{k}\left(\widehat{Y_{k} H_{k}}-\tilde{\mathcal{K}} \hat{Y}_{k}\right)+\chi_{k} \hat{Y}_{k}, \quad \tilde{\mathcal{K}}=\frac{\hat{u}^{2}+\hat{v}^{2}}{2} \text {, } \\
& \tilde{V}=\kappa_{1} \widetilde{v Y_{1}}+\kappa_{2} \widetilde{v Y_{2}} \text {. }
\end{aligned}
$$

\section{Appendix C. Mathematical expression of the relaxed variables}

We report here the mathematical formulas used to compute the equilibrium variables in the stiff relaxation procedures of Sections 3.3, 4.1, and 4.2 for the particular case of the stiffened gas equation of state.

\section{C.1. Mechanical relaxation}

The following quadratic equation can be obtained for the relaxed pressure $p^{*}$ at mechanical equilibrium (see Section 3.3):

$$
a\left(p^{*}\right)^{2}+b p^{*}+d=0
$$

with the coefficients $a, b$, and $d$ defined by

$$
\begin{aligned}
& a=1+\gamma_{2} \alpha_{1}^{0}+\gamma_{1} \alpha_{2}^{0} \\
& b=C_{1} \alpha_{2}^{0}+C_{2} \alpha_{1}^{0}-\left(1+\gamma_{2}\right) \alpha_{1}^{0} p_{1}^{0}-\left(1+\gamma_{1}\right) \alpha_{2}^{0} p_{2}^{0}, \\
& d=-\left(C_{2} \alpha_{1}^{0} p_{1}^{0}+C_{1} \alpha_{2}^{0} p_{2}^{0}\right)
\end{aligned}
$$

together with $C_{1}=2 \gamma_{1} \pi_{1}+\left(\gamma_{1}-1\right) p_{\mathrm{I}}^{0}$ and $C_{2}=2 \gamma_{2} \pi_{2}+\left(\gamma_{2}-1\right) p_{\mathrm{I}}^{0}$. This gives the solution

$$
p^{*}=\frac{-b+\sqrt{b^{2}-4 a d}}{2 a}
$$

where we have retained the root corresponding to a physically meaningful positive value of the pressure (note that $C_{1}, C_{2}>$ $0, a>0, d<0$ and $b^{2}-4 a d>0$ ). The values of the volume fraction $\alpha_{1}^{*}$ at equilibrium are then found as

$$
\alpha_{1}^{*}=\frac{\left(\gamma_{1}-1\right) p^{*}+2 p_{1}^{0}+C_{1}}{\left(\gamma_{1}+1\right) p^{*}+C_{1}} \alpha_{1}^{0} .
$$




\section{C.2. Thermal relaxation}

From the invariance conditions (42) and the equilibrium conditions (43) we can derive a single quadratic equation to be solved for the pressure $p^{* *}$ at mechanical and thermal equilibrium:

$$
a^{\prime}\left(p^{* *}\right)^{2}+b^{\prime} p^{* *}+d^{\prime}=0
$$

with the coefficients $a^{\prime}, b^{\prime}$, and $d^{\prime}$ defined by

$$
\begin{aligned}
a^{\prime}= & C_{v 1}\left(\alpha_{1} \rho_{1}\right)^{0}+C_{v 2}\left(\alpha_{2} \rho_{2}\right)^{0}, \\
b^{\prime}= & \eta_{1} C_{v 1}\left(\gamma_{1}-1\right)\left(\left(\alpha_{1} \rho_{1}\right)^{0}\right)^{2}+\eta_{2} C_{v 2}\left(\gamma_{2}-1\right)\left(\left(\alpha_{2} \rho_{2}\right)^{0}\right)^{2}+\left(\alpha_{1} \rho_{1}\right)^{0} C_{v 1}\left(\gamma_{1} \pi_{1}+\pi_{2}\right) \\
& +\left(\alpha_{2} \rho_{2}\right)^{0} C_{v 2}\left(\gamma_{2} \pi_{2}+\pi_{1}\right)+\left(\alpha_{1} \rho_{1}\right)^{0}\left(\alpha_{2} \rho_{2}\right)^{0}\left(\eta_{1} C_{v 2}\left(\gamma_{2}-1\right)+\eta_{2} C_{v 1}\left(\gamma_{1}-1\right)\right) \\
& -\mathcal{E}^{0}\left(C_{v 1}\left(\gamma_{1}-1\right)\left(\alpha_{1} \rho_{1}\right)^{0}+C_{v 2}\left(\gamma_{2}-1\right)\left(\alpha_{2} \rho_{2}\right)^{0}\right), \\
d^{\prime}= & \eta_{1} C_{v 1}\left(\gamma_{1}-1\right) \pi_{2}\left(\left(\alpha_{1} \rho_{1}\right)^{0}\right)^{2}+\eta_{2} C_{v 2}\left(\gamma_{2}-1\right) \pi_{1}\left(\left(\alpha_{2} \rho_{2}\right)^{0}\right)^{2}+\left(\left(\alpha_{1} \rho_{1}\right)^{0} C_{v 1} \gamma_{1}\right. \\
& \left.+\left(\alpha_{2} \rho_{2}\right)^{0} C_{v 2} \gamma_{2}\right) \pi_{2} \pi_{1}+\left(\alpha_{1} \rho_{1}\right)^{0}\left(\alpha_{2} \rho_{2}\right)^{0}\left(\eta_{1} C_{v 2}\left(\gamma_{2}-1\right) \pi_{1}+\eta_{2} C_{v 1}\left(\gamma_{1}-1\right) \pi_{2}\right) \\
& -\mathcal{E}^{0}\left(C_{v 1}\left(\gamma_{1}-1\right) \pi_{2}\left(\alpha_{1} \rho_{1}\right)^{0}+C_{v 2}\left(\gamma_{2}-1\right) \pi_{1}\left(\alpha_{2} \rho_{2}\right)^{0}\right) .
\end{aligned}
$$

Having obtained $p^{* *}$ from the above equation, the equilibrium volume fraction is then

$$
\alpha_{1}^{* *}=\frac{C_{v 1}\left(\gamma_{1}-1\right)\left(p^{* *}+\pi_{2}\right)\left(\alpha_{1} \rho_{1}\right)^{0}}{C_{v 1}\left(\gamma_{1}-1\right)\left(p^{* *}+\pi_{2}\right)\left(\alpha_{1} \rho_{1}\right)^{0}+C_{v 2}\left(\gamma_{2}-1\right)\left(p^{* *}+\pi_{1}\right)\left(\alpha_{2} \rho_{2}\right)^{0}},
$$

and the equilibrium temperature is

$$
T^{* *}=\frac{\left(p^{* *}+\pi_{1}\right) \alpha_{1}^{* *}}{\left(\gamma_{1}-1\right) C_{v 1}\left(\alpha_{1} \rho_{1}\right)^{0}}=\frac{\left(p^{* *}+\pi_{2}\right)\left(1-\alpha_{1}\right)^{* *}}{\left(\gamma_{2}-1\right) C_{v 2}\left(\alpha_{2} \rho_{2}\right)^{0}} .
$$

\section{C.3. Thermo-chemical relaxation}

By using the conservation conditions (46) and the equilibrium conditions (47), together with the relations $\mathcal{E}_{k}=\mathcal{E}_{k}\left(p_{k}, \rho_{k}\right)$, we can first obtain a quadratic equation for the equilibrium temperature as a function of the equilibrium pressure, $T^{\circledast}=$ $T^{\circledast}\left(p^{\circledast}\right)$. We have:

$$
a_{p}\left(p^{\circledast}\right)\left(T^{\circledast}\right)^{2}+b_{p}\left(p^{\circledast}\right) T^{\circledast}+d_{p}\left(p^{\circledast}\right)=0,
$$

where the coefficients $a_{p}\left(p^{\circledast}\right), b_{p}\left(p^{\circledast}\right)$, and $d_{p}\left(p^{\circledast}\right)$ are

$$
\begin{aligned}
a_{p}\left(p^{\circledast}\right)= & \rho^{0} C_{v 1} C_{v 2}\left(\left(\gamma_{2}-1\right)\left(p^{\circledast}+\gamma_{1} \pi_{1}\right)-\left(\gamma_{1}-1\right)\left(p^{\circledast}+\gamma_{2} \pi_{2}\right)\right), \\
b_{p}\left(p^{\circledast}\right)= & \mathcal{E}^{0}\left(\left(\gamma_{1}-1\right) C_{v 1}\left(p^{\circledast}+\pi_{2}\right)-\left(\gamma_{2}-1\right) C_{v 2}\left(p^{\circledast}+\pi_{1}\right)\right) \\
& +\rho^{0}\left(\left(\gamma_{2}-1\right) C_{v 2} \eta_{1}\left(p^{\circledast}+\pi_{1}\right)-\left(\gamma_{1}-1\right) C_{v 1} \eta_{2}\left(p^{\circledast}+\pi_{2}\right)\right) \\
& +C_{v 2}\left(p^{\circledast}+\pi_{1}\right)\left(p^{\circledast}+\gamma_{2} \pi_{2}\right)-C_{v 1}\left(p^{\circledast}+\pi_{2}\right)\left(p^{\circledast}+\gamma_{1} \pi_{1}\right), \\
d_{p}\left(p^{\circledast}\right)= & \left(\eta_{2}-\eta_{1}\right)\left(p^{\circledast}+\pi_{1}\right)\left(p^{\circledast}+\pi_{2}\right) .
\end{aligned}
$$

This gives:

$$
T^{\circledast}\left(p^{\circledast}\right)=\frac{-b_{p}\left(p^{\circledast}\right)+\sqrt{\left(b_{p}\left(p^{\circledast}\right)\right)^{2}-4 a_{p}\left(p^{\circledast}\right) d_{p}\left(p^{\circledast}\right)}}{2 a_{p}\left(p^{\circledast}\right)} .
$$

By introducing this relation in the Gibbs free energy equilibrium condition (47c) (see also (11)) we finally obtain a single equation for the pressure $p^{\circledast}$ at mechanical and thermo-chemical equilibrium:

$$
A_{\mathrm{S}}+\frac{B_{\mathrm{S}}}{T^{\circledast}\left(p^{\circledast}\right)}+C_{\mathrm{S}} \log T^{\circledast}\left(p^{\circledast}\right)+D_{\mathrm{S}} \log \left(p^{\circledast}+\pi_{1}\right)-\log \left(p^{\circledast}+\pi_{2}\right)=0,
$$

with $A_{\mathrm{S}}, B_{\mathrm{S}}, C_{\mathrm{S}}$, and $C_{\mathrm{S}}$ as in (11b). Once we compute $p^{\circledast}$, the equilibrium temperature $T^{\circledast}$ can be obtained from (C.7), and the values of the equilibrium densities and volume fractions are given by the following relations:

$$
\rho_{k}^{\circledast}=\frac{p^{\circledast}+\pi_{k}}{\left(\gamma_{k}-1\right) C_{v k} T^{\circledast}}, \quad k=1,2, \quad \alpha_{1}^{\circledast}=\frac{\rho^{*}-\rho_{2}^{\circledast}}{\rho_{1}^{\circledast}-\rho_{2}^{\circledast} .}
$$


As a final remark, note that the solution of the system (46)-(47) ( $p T g$ equilibrium solution) for full thermodynamic equilibrium may not be physically admissible. In such a case we consider that the mixture is composed nearly of the species $k$ that has the highest entropy $s_{k}^{* *}$, by using an idea similar to [14]. Therefore, we fix the value of $\alpha_{k}^{\circledast}=\bar{\alpha}_{k}^{\circledast}=1-\epsilon$ (for instance $\epsilon=10^{-8}$ ). Then, we obtain an algebraic system for the unknowns $p^{\circledast}, T^{\circledast}, \rho_{1}^{\circledast}, \rho_{2}^{\circledast}$ by using the conservation conditions (46) and the pressure and temperature equilibrium conditions (47a) and (47b). Again, for the SG EOS, this system can be reduced to a single quadratic equation for $p^{\circledast}$ (or for $T^{\circledast}$ ). We select the physically admissible solution of this quadratic equation that maximizes the total entropy $s^{\circledast}=Y_{1}^{\circledast} s_{1}^{\circledast}+Y_{2}^{\circledast} s_{2}^{\circledast}$.

\section{References}

[1] C.E. Brennen, Fundamentals of Multiphase Flow, Cambridge University Press, 2005.

[2] J.-P. Franc, J.-M. Michel, Fundamentals of Cavitation, Springer Science+Business Media, Dordrecht, 2005.

[3] P.V. Carey, Liquid-Vapor Phase-Change Phenomena, Taylor and Francis, 1992.

[4] T.G. Liu, B.C. Khoo, W.F. Xie, Isentropic one-fluid modeling of unsteady cavitating flow, J. Comput. Phys. 201 (2004) 80-108.

[5] W.F. Xie, T.G. Liu, B.C. Khoo, Application of a one-fluid model for large scale homogeneous unsteady cavitation: The modified Schmidt model, Comput. Fluids 35 (2006) 1177-1192.

[6] J.R. Edwards, R.K. Franklin, M.-S. Liou, Low-diffusion flux-splitting methods for real fluid flows with phase transitions, AIAA J. 38 (2000) $1624-1633$.

[7] D. Jamet, O. Lebaigue, N. Coutris, J.M. Delhaye, The second gradient method for the direct numerical simulation of liquid-vapor flows with phase change, J. Comput. Phys. 169 (2001) 624-651.

[8] R. Saurel, F. Petitpas, R. Abgrall, Modelling phase transition in metastable liquids: application to cavitating and flashing flows, J. Fluid Mech. 607 (2008) 313-350.

[9] R. Saurel, F. Petitpas, R.A. Berry, Simple and efficient relaxation methods for interfaces separating compressible fluids, cavitating flows and shocks in multiphase mixture, J. Comput. Phys. 228 (2009) 1678-1712.

[10] R. Saurel, O. Le Métayer, A multiphase model for compressible flows with interfaces, shocks, detonation waves and cavitation, J. Fluid Mech. 431 (2001) 239-271.

[11] Y. Utturkar, J. Wu, G. Wang, W. Shyy, Recent progress in modeling of cryogenic cavitation for liquid rocket propulsion, Prog. Aerosp. Sci. 41 (2005) 558-608.

[12] T. Barberon, P. Helluy, Finite volume simulation of cavitating flows, Comput. Fluids 34 (2005) 832-858.

[13] S. Müller, M. Bachmann, D. Kröninger, T. Kurz, P. Helluy, Comparison and validation of compressible flow simulations of laser-induced cavitation bubbles, Comput. Fluids 38 (2009) 1850-1862.

[14] G. Faccanoni, S. Kokh, G. Allaire, Modelling and simulation of liquid-vapor phase transition in compressible flows based on thermodynamical equilibrium, Modél. Math. Anal. Numér. 46 (2012) 1029-1054.

[15] K.-M. Shyue, An adaptive moving-mesh relaxation scheme for compressible two-phase barotropic flow with cavitation, in: Proceedings of ASME-JSMEKSME Joint Fluids Engineering Conference 2011, ASME, 2011, pp. AJK2011-AJK04009.

[16] M.R. Baer, J.W. Nunziato, A two-phase mixture theory for the deflagration-to-detonation transition (DDT) in reactive granular materials, Int. J. Multiph. Flow 12 (1986) 861-889.

[17] A. Zein, M. Hantke, G. Warnecke, Modeling phase transition for compressible two-phase flows applied to metastable liquids, J. Comput. Phys. 229 (2010) 2964-2998.

[18] A. Kapila, R. Menikoff, J.B. Bdzil, S.F. Son, D. Stewart, Two-phase modeling of deflagration-to-detonation transition in granular materials: Reduced equations, Phys. Fluids 13 (2001) 3002-3024.

[19] R.J. LeVeque, Finite Volume Methods for Hyperbolic Problems, Cambridge University Press, 2002.

[20] R.J. LeVeque, M.J. Berger, cLAwPACK software version 4.5, http://www.clawpack.org, 2011.

[21] R. Saurel, R. Abgrall, A multiphase Godunov method for compressible multifluid and multiphase flows, J. Comput. Phys. 150 (1999) $425-467$.

[22] F. Petitpas, J. Massoni, R. Saurel, E. Lapebie, L. Munier, Diffuse interface model for high speed cavitating underwater systems, Int. J. Multiph. Flow 35 (2009) 747-759.

[23] S. Le Martelot, B. Nkonga, R. Saurel, Liquid and liquid-gas flows at all speeds: Reference solutions and numerical schemes, INRIA Research Report N. 7935, 2012.

[24] A. Murrone, H. Guillard, A five equation reduced model for compressible two phase flow problems, J. Comput. Phys. 202 (2005) 664-698.

[25] A.B. Wood, A Textbook of Sound, G. Bell and Sons Ltd., London, 1930.

[26] F. Petitpas, E. Franquet, R. Saurel, O. Le Métayer, A relaxation-projection method for compressible flows. Part II: Artificial heat exchanges for multiphase shocks, J. Comput. Phys. 225 (2007) 2214-2248.

[27] H.B. Callen, Thermodynamics and an Introduction to Thermostatistics, John Wiley \& Sons, 1985.

[28] G. Allaire, S. Clerc, S. Kokh, A five-equation model for the simulation of interfaces between compressible fluids, J. Comput. Phys. 181 (2002) $577-616$.

[29] N. Andrianov, G. Warnecke, The Riemann problem for the Baer-Nunziato two-phase flow model, J. Comput. Phys. 195 (2004) $434-464$.

[30] D.W. Schwendeman, C.W. Wahle, A.K. Kapila, The Riemann problem and a high-resolution Godunov method for a model of compressible two-phase flow, J. Comput. Phys. 212 (2006) 490-526.

[31] S.A. Tokareva, E.F. Toro, HLLC-type Riemann solver for the Baer-Nunziato equations of compressible two-phase flow, J. Comput. Phys. 229 (2010) 3573-3604.

[32] Y. Jinbo, T. Ogasawara, H. Takahira, Numerical investigation of nonspherical bubble collapse near boundaries by the improved ghost fluid method, in: C.-D. Ohl, E. Klaseboer, S.W. Ohl, S.W. Gong, B.C. Khoo (Eds.), Proceedings of Eighth Int. Symp. on Cavitation (CAV 2012), Research Publishing Services, 2012.

[33] T. Flåtten, H. Lund, Relaxation two-phase models and the subcharacteristic condition, Math. Models Methods Appl. Sci. 21 (2011) $2379-2407$.

[34] H. Lund, A hierarchy of relaxation models for two-phase flows, SIAM J. Appl. Math. 72 (2012) 1713-1741.

[35] T.P. Liu, Hyperbolic conservation laws with relaxation, Commun. Math. Phys. 108 (1987) 153-175.

[36] O. Le Métayer, J. Massoni, R. Saurel, Elaborating equations of state of a liquid and its vapor for two-phase flow models, Int. J. Therm. Sci. 43 (2004) $265-276$.

[37] O. Le Métayer, J. Massoni, R. Saurel, Modeling evaporation fronts with reactive Riemann solvers, J. Comput. Phys. 205 (2005) 567-610.

[38] E.F. Toro, Riemann Solvers and Numerical Methods for Fluid Dynamics, Springer-Verlag, Berlin, Heidelberg, 1997.

[39] R.J. LeVeque, Wave propagation algorithms for multi-dimensional hyperbolic systems, J. Comput. Phys. 131 (1997) 327-353.

[40] S.K. Godunov, A difference method for numerical calculation of discontinuous solutions of the equations of hydrodynamics, Mat. Sb. 47 (1959) $271-306$.

[41] E. Godlewski, P.-A. Raviart, Numerical Approximation of Hyperbolic Systems of Conservation Laws, Springer-Verlag, New York, 1996.

[42] D. Bale, R.J. LeVeque, S. Mitran, J.A. Rossmanith, A wave-propagation method for conservation laws and balance laws with spatially varying flux functions, SIAM J. Sci. Comput. 24 (2002) 955-978. 
[43] A. Harten, P.D. Lax, B. van Leer, On upstream differencing and Godunov-type schemes for hyperbolic conservation laws, SIAM Rev. 25 (1983) 35-61.

[44] E.F. Toro, M. Spruce, W. Speares, Restoration of the contact surface in the HLL Riemann solver, Shock Waves 4 (1994) $25-34$.

[45] P.L. Roe, Approximate Riemann solvers, parameter vectors, and difference schemes, J. Comput. Phys. 43 (1981) 357-372.

[46] G.D. Maso, P.G. LeFloch, F. Murat, Definition and weak stability of nonconservative products, J. Math. Pures Appl. 74 (1995) $483-548$.

[47] C. Parés, Numerical methods for nonconservative hyperbolic systems: a theoretical framework, SIAM J. Numer. Anal. 44 (2006) 300-321.

[48] M.J. Castro, P. LeFloch, M.L. Muñoz-Ruiz, C. Parés, Why many theories of shock waves are necessary: Convergence error in formally path-consistent schemes, J. Comput. Phys. 227 (2008) 8107-8129.

[49] R. Abgrall, S. Karni, A comment on the computation of non-conservative products, J. Comput. Phys. 229 (2010) $2759-2763$.

[50] S.F. Davis, Simplified second-order Godunov-type methods, SIAM J. Sci. Stat. Comput. 9 (1988) 445-473.

[51] M. Pelanti, K.-M. Shyue, A mixture-energy-consistent numerical approximation of a two-phase flow model for fluids with interfaces and cavitation, in: Proceedings of the Fourteenth International Conference on Hyperbolic Problems: Theory, Numerics, Applications, Padua, Italy, June 25-29, 2012, AIMS, submitted for publication.

[52] K.-M. Shyue, A high-resolution mapped grid algorithm for compressible multiphase flow problems, J. Comput. Phys. 229 (2010) $8780-8801$.

[53] F. Caro, F. Coquel, D. Jamet, S. Kokh, A simple finite-volume method for compressible isothermal two-phase flows simulation, Int. J. Finite Vol. 6 (1) (2006).

[54] R. Saurel, N. Favrie, F. Petitpas, M.-H. Lallemand, S.L. Gavrilyuk, Modelling dynamic and irreversible powder compaction, J. Fluid Mech. 664 (2010) 348-396.

[55] H. Guillard, C. Viozat, On the behavior of upwind schemes in the low Mach number limit, Comput. Fluids 28 (1999) 63-86.

[56] H. Guillard, A. Murrone, On the behavior of upwind schemes in the low Mach number limit: II. Godunov-type schemes, Comput. Fluids 338 (2004) 655-675.

[57] M. Bilanceri, F. Beux, M.V. Salvetti, An implicit low-diffusive HLL scheme with complete time linearization: Application to cavitating barotropic flows, Comput. Fluids 39 (2010) 1990-2006.

[58] B. Braconnier, J.-J. Hu, Y.-Y. Niu, B. Nkonga, K.-M. Shyue, Numerical simulations of low Mach compressible two-phase flows: Preliminary assessment of some basic solution techniques, ESAIM Proc. 28 (2009) 117-134.

[59] R. Abgrall, H. Kumar, Numerical approximation of a compressible multiphase system, Commun. Comput. Phys., http://dx.doi.org/10.4208/cicp. 110313.230913a, in press. 